\title{
The General Circulation of the Atmosphere
}

\section{Tapio Schneider}

California Institute of Technology, Pasadena, California 91125; email: tapio@caltech.edu

\author{
Annu. Rev. Earth Planet. Sci. \\ 2006. 34:655-88 \\ The Annual Review of \\ Earth and Planetary Science \\ is online at \\ earth.annualreviews.org \\ doi: $10.1146 /$ \\ annurev.earth.34.031405.125144 \\ Copyright (c) 2006 by \\ Annual Reviews. All rights \\ reserved \\ 0084-6597/06/0530- \\ $0655 \$ 20.00$
}

\section{Key Words}

Hadley circulation, macroturbulence, baroclinic eddies, jets, scaling laws

\begin{abstract}
Theories of how Earth's surface climate may change in the future, of how it may have been in the past, and of how it is related to climates of other planets must build upon a theory of the general circulation of the atmosphere. The view of the atmospheric general circulation presented here focuses not on Earth's general circulation as such but on a continuum of idealized circulations with axisymmetric flow statistics. Analyses of observational data for Earth's atmosphere, simulations with idealized general circulation models, and theoretical considerations suggest how characteristics of the tropical Hadley circulation, of the extratropical circulation, and of atmospheric macroturbulence may depend on parameters such as the planet radius and rotation rate and the strength of the differential heating at the surface.
\end{abstract}




\section{INTRODUCTION}

In the mean, zonal surface winds on Earth are easterly (westward) in low latitudes, westerly (eastward) in midlatitudes, and easterly or nearly vanishing in high latitudes. The strength of the mean zonal surface wind varies seasonally, but the pattern of alternating easterlies and westerlies is present throughout the year, with slight seasonal shifts of the latitudes at which the mean zonal surface wind changes sign (Figure $\mathbf{1} b$ shows January as an example). The mean meridional surface wind is weaker than the mean zonal surface wind. It is directed poleward in regions of surface westerlies and equatorward in regions of surface easterlies. In boreal summer, the monsoons of the Northern Hemisphere lead to a mean northward surface wind across the equator, which typically has a westerly component in monsoon regions.

That mean surface winds have definite directions has been exploited in centuries past by navigators, who called winds with a prevalent direction trade winds, a term we now use more restrictively to denote the tropical easterly winds. "The Causes of the
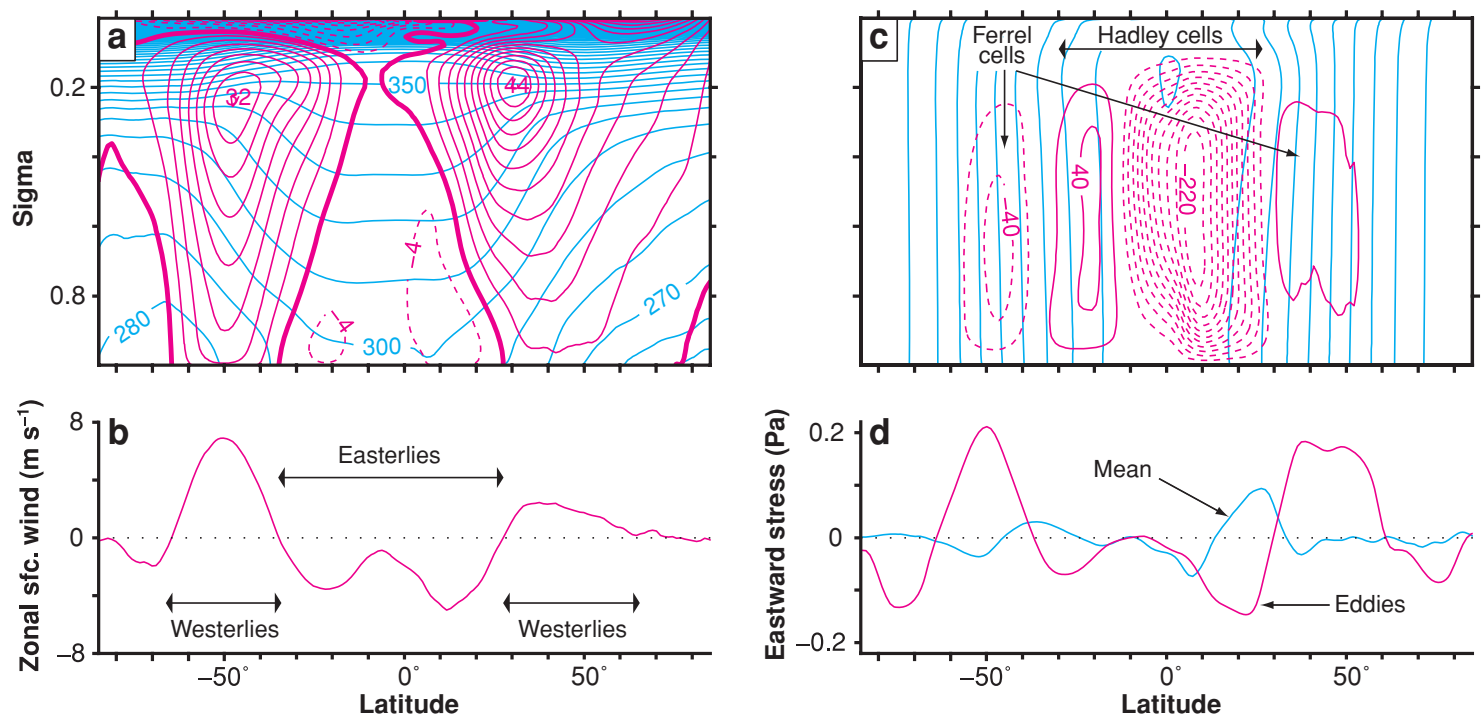

Figure 1

Temporal and zonal mean circulation statistics for January according to reanalysis data for the years 1980-2001 (Kållberg et al. 2004). (a) Zonal wind (magenta) and potential temperature (light blue). Contour intervals are $4 \mathrm{~m} \mathrm{~s}^{-1}$ for zonal wind and $10 \mathrm{~K}$ for potential temperature. The thick magenta line is the zero zonal wind contour. (b) Zonal wind at the surface. (c) Eulerian mass flux streamfunction (magenta) and angular momentum (light blue). Contour intervals are $20 \times 10^{9} \mathrm{~kg} \mathrm{~s}^{-1}$ for streamfunction and $0.1 \Omega a^{2}$ for angular momentum, with angular momentum decreasing monotonically from the equator to the poles. Negative streamfunction values (dashed contours) correspond to clockwise rotation, positive values (solid contours) to counterclockwise rotation. (d) Vertically integrated momentum flux convergence (eastward stress) due to mean circulations (light blue) and due to eddies (magenta), with eddies defined as fluctuations about the temporal and zonal mean. The vertical coordinate in $(a)$ and (c) is $\sigma=p / p_{\mathrm{s}}$ (pressure $p$ normalized by surface pressure $p_{\mathrm{s}}$ ). 
General Trade-Winds have not been fully explained by any of those who have wrote on that Subject," Hadley wrote in 1735, "for want of more particularly and distinctly considering the Share the diurnal Motion of Earth has in the Production of them." Hadley recognized that Earth's rotation is responsible for the direction of the mean surface winds and that a global circulation is necessary to maintain them.

According to Hadley, if one corrects minor shortcomings of his argumentation (Thomson 1892, Lorenz 1967), differential heating causes air to rise near the equator and, by conservation of mass, to flow poleward aloft. Hadley envisaged a steady circulation symmetric about Earth's rotation axis. Absent frictional forces, each parcel of poleward flowing air in the axisymmetric circulation conserves its absolute angular momentum about Earth's axis, $M=(\Omega a \cos \phi+u) a \cos \phi$ per unit mass in the approximation of the atmosphere as a thin spherical shell, with radius $a$, angular velocity of planetary rotation $\Omega$, latitude $\phi$, and eastward wind component $u$ (Peixoto \& Oort 1992, Chapter 11). As an air parcel flows poleward, its distance to Earth's axis $a \cos \phi$ decreases, so that angular momentum conservation implies that the parcel's eastward wind component $u$ increases. ${ }^{1}$ Differential cooling causes air to sink in higher latitudes, again under conservation of angular momentum of air parcels, and thus leads to surface westerlies in higher latitudes. The surface winds are retarded by surface drag and are deflected equatorward, to provide the return flow necessary to close the circulation. Because of surface drag, the equatorward return flow does not conserve angular momentum, but if one reverses the arguments for the poleward flow in the upper branch of the circulation, it does, according to Hadley, give rise to surface easterlies in lower latitudes, albeit weaker ones than angular momentum conservation would imply. Because Earth's spin angular momentum is nearly conserved (tidal and other external torques can be neglected in theories of the circulation of the lower atmosphere), the eastward drag the surface westerlies exert on Earth's surface in higher latitudes must be balanced by the westward drag the surface easterlies exert on Earth's surface in lower latitudes for Earth's rotation rate to remain constant. According to this theory, a thermally direct meridional circulation in each hemisphere transports angular momentum from low latitudes, where the drag on surface easterlies transfers angular momentum from the surface to the atmosphere, to high latitudes, where the drag on surface westerlies transfers angular momentum from the atmosphere to the surface. The circulation also transports heat from low to high latitudes.

In the nineteenth century, new observations showed that midlatitude surface westerlies have, in the mean, a poleward component, instead of the equatorward component demanded by Hadley's theory. To account for the new observations, Thomson $(1857,1892)$ and Ferrel (1859) amended Hadley's theory by positing secondary, thermally indirect meridional circulation cells in midlatitudes. The indirect circulation cells were thought to be shallow, embedded under Hadley's hemispheric circulation cells, with, in today's terms, the Coriolis force on the poleward surface wind component approximately balancing the drag on the surface westerlies (Ekman balance).

\footnotetext{
${ }^{1}$ Hadley actually thought that absolute zonal velocity rather than absolute angular momentum was conserved, a conceptual but merely quantitative error in his qualitative argumentation (Thomson 1892, Lorenz 1967).
} 
General circulation: totality of mean large-scale motions of atmosphere

Hadley cell: thermally direct tropical circulation cell

Ferrel cell: thermally indirect midlatitude circulation cell

Macroturbulence: totality of irregular motions of large-scale eddies, characterized by small Rossby number and small aspect ratio
But the axisymmetric general circulation theories of Hadley and his successors turned out to be untenable when Defant (1921), Jeffreys (1926), and others realized that large-scale eddies-cyclones and anticyclones with length scales of order $1000 \mathrm{~km}$ - are not perturbations of an idealized axisymmetric circulation but are essential, in particular, for the atmospheric transport of heat and angular momentum [see Lorenz $(1967,1983)$ for an historical overview]. The thermally direct meridional circulation named after Hadley extends from equatorial regions only to about $30^{\circ}$ latitude in each hemisphere (Figure 1c), with slight seasonal shifts of the poleward boundaries of the Hadley cells. The thermally indirect midlatitude cells named after Ferrel cannot be understood as axisymmetric circulations. Angular momentum is not conserved along streamlines of the Ferrel cells. Horizontal eddy momentum fluxes make it possible that streamlines in the upper branches of the Ferrel cells cross angular momentum contours (Figure 1 c). The Coriolis force on the equatorward mean flow in the upper branches of the Ferrel cells approximately balances a convergence of horizontal eddy momentum fluxes. Because horizontal eddy momentum fluxes and momentum advection by the mean flow are weak near the surface, the Coriolis forces on the mean meridional surface winds in the Ferrel and Hadley cells approximately balance the drag on the mean zonal surface winds, as implied in the axisymmetric theories of Ferrel and Thomson. Hence, the mean meridional surface wind is generally directed poleward in regions of surface westerlies and equatorward in regions of surface easterlies. The angular momentum transfered from the atmosphere to the surface by the drag on midlatitude westerlies is transported into midlatitudes not primarily by the mean meridional flow but by eddies, whose angular momentum transport from high and low latitudes into midlatitudes implies eastward stresses on Earth's surface in midlatitudes and westward stresses in low and high latitudes (Figure 1d). The divergence of eddy momentum fluxes in high latitudes, concentrated in the upper troposphere, and the drag on any high-latitude surface easterlies are approximately balanced by Coriolis forces on the mean meridional flow in weak, thermally direct polar circulation cells (not visible in Figure 1c). Instead of Hadley's hemispheric circulation cells extending from equatorial regions to high latitudes, there are thus three circulation cells in each hemisphere, at least two of which are due to eddy fluxes.

A theory of the general circulation of the atmosphere must build upon a theory of the macroturbulence consisting of large-scale eddies. Eddies generated by baroclinic instability generally transport angular momentum into the latitude zones in which they are generated (Held 1975, 2000, Simmons \& Hoskins 1978, Ioannou \& Lindzen 1986). In a statistically steady state, the angular momentum flux into or out of an atmospheric column is balanced by surface drag, so surface westerlies appear in baroclinic zones into which eddies transport angular momentum. The vertical structure of the winds and the strength of upper-level jets are connected to the surface winds through thermal-wind (or gradient-wind) balance, which relates the vertical shear of the zonal wind to meridional temperature (or potential temperature) gradients, as seen in Figure 1a. [See Holton (2004) for a discussion of thermal-wind and gradientwind balance and other basic concepts of atmospheric dynamics.] The meridional 
temperature gradients, in turn, are determined by a balance of radiative processes, ocean circulations, and meridional fluxes of latent and sensible heat, fluxes that, in midlatitudes, are primarily effected by eddies. Particularly in low latitudes, the mean meridional circulations also contribute to the fluxes of angular momentum, heat, and water vapor, transporting, for example, angular momentum from equatorial regions into the subtropics, from where eddies transport it into midlatitudes (Figure 1d). A minimal mean-field theory of the general circulation of the atmosphere, then, must account quantitatively for the mutual dependence of eddy fluxes and mean fields such as mean meridional temperature gradients. We do not have such a theory.

I review our present understanding of the general circulation of the atmosphere, focusing not on Earth's atmosphere as such but on idealized atmospheres with axisymmetric boundary conditions-with incoming solar radiation only depending on latitude and with a homogeneous surface - atmospheres that have axisymmetric circulation statistics. Although the zonal-mean effects of statistically nonaxisymmetric circulation features such as monsoons or stationary waves are significant, a general circulation theory for idealized atmospheres with axisymmetric circulation statistics is a prerequisite for any future, more complete, general circulation theory, which must be reducible to this canonical case. Present-day computational resources allow us to simulate such atmospheres with idealized general circulation models (GCMs) numerically, not only in the circulation regime comprising the seasonal cycle and recent climatic variations of Earth's atmosphere but over a broad continuum of possible planetary circulations, of which circulations resembling that of present-day Earth represent a part. Investigations of such a circulation continuum are indispensable if one wants to infer macroscopic laws governing atmospheric circulations and wants to understand circulation changes that may have occurred in the past or may occur in the future and that go beyond those for which we have detailed observational data. Comparisons with the wealth of observational data for Earth's atmosphere, together with the much sparser data for other planetary circulations, can provide insurances that inferences drawn from simulations are empirically relevant. Unlike previous generations of atmospheric dynamicists, we are no longer limited by sparseness of observational data or insufficient computational resources to complete the project of developing a quantitative theory of the general circulation at least of idealized atmospheres.

I discuss the tropical Hadley circulation and the influence macroturbulence may have on it in Section 2 and the extratropical circulation and aspects of atmospheric macroturbulence in Section 3. Moist processes such as moist convection and latent heat release in phase changes of water are largely sidelined in the discussions, not because they would be unimportant- they generally are important—but to draw attention to unresolved fundamental questions about the general circulation of dry atmospheres, questions whose resolution is a prerequisite for any future general circulation theory, moist or dry. I focus on the general circulation of the troposphere because it is primarily responsible for the maintenance and variability of the surface climate. 


\section{THE TROPICAL HADLEY CIRCULATION}

Hide's theorem: in steady axisymmetric circulations, zonal wind cannot exceed angular momentumconserving zonal wind

\subsection{Nearly Inviscid Axisymmetric Circulations}

In idealized atmospheres with axisymmetric boundary conditions, absent nonaxisymmetric perturbations, a circulation that is initially axisymmetric remains axisymmetric, albeit not necessarily steady. So we can consider theoretically and simulate numerically axisymmetric circulations in idealized atmospheres, postponing questions of the effects on these circulations of instabilities with respect to nonaxisymmetric perturbations. We examine what Lorenz (1969) called the "ideal Hadley circulation," essentially the circulation envisaged by Hadley.

A steady axisymmetric circulation in which angular momentum disperses diffusively, however weak the diffusion, cannot have an extremum of absolute angular momentum away from boundaries, which constrains zonal winds to be weaker than or at most equal to the zonal winds implied by an angular momentum-conserving circulation (Hide 1969, Schneider 1977). If there were an angular momentum extremum away from boundaries, there would exist a closed contour of constant angular momentum surrounding it in the meridional plane. The advective flux of angular momentum across the contour would vanish because, in a steady state, the mass flux across the contour would vanish. But diffusion of angular momentum-typically, vertical diffusion representing small-scale turbulent fluxes-implies that there would be a downgradient flux of angular momentum across the contour, which could not be balanced, so angular momentum extrema away from boundaries are impossible. Angular momentum must attain its maximum value in a region of easterlies at the surface, where the surface drag on the easterlies transfers angular momentum from the surface to the atmosphere immediately above it, from where it can diffuse into the interior atmosphere. The angular momentum per unit mass $M=(\Omega a \cos \phi+u) a \cos \phi$ must therefore be everywhere less than or equal to $\Omega a^{2}$, and the zonal winds must be less than or equal to the angular momentum-conserving zonal wind $u_{M}=\Omega a \sin ^{2} \phi / \cos \phi$ corresponding to $M=\Omega a^{2}$, a result known as Hide's theorem. A steady state with stronger eastward winds-for example, equatorial westerlies (superrotation) such as seen on the giant planets-can only be maintained if diffusion of angular momentum is balanced by upgradient eddy fluxes of angular momentum.

If the atmosphere were inviscid, radiative (or radiative-convective) equilibrium without a meridional circulation would be a steady axisymmetric solution of the equations of motion. But if angular momentum disperses diffusively, radiative equilibrium may not satisfy Hide's theorem. Suppose that, above a planetary boundary layer, angular momentum diffusion and frictional forces are weak, hydrostatic balance holds, and meridional winds are much weaker than zonal winds, as is generally the case in Earth's atmosphere. The meridional momentum equation can then be approximated by gradient-wind balance

$$
f u+\frac{\tan \phi}{a} u^{2} \approx-\frac{1}{a} \frac{\partial \Phi}{\partial \phi},
$$

where $f=2 \Omega \sin \phi$ is the Coriolis parameter (vertical component of planetary vorticity), $\Phi=g z$ the geopotential, and the meridional derivative is a derivative at constant 
pressure. If we substitute Hide's result $u \leq u_{M}$ and make the small-angle approximation $\phi \ll 1$ (adequate in the Tropics), we find $-\partial_{\phi} \Phi \lesssim 2 \Omega^{2} a^{2} \phi^{3}$. In the Tropics, above the planetary boundary layer and independent of surface boundary conditions, geopotential can decrease at most quartically with latitude. Because the geopotential at pressure $p$, according to the hydrostatic relation $\Phi(p)=-R\langle T\rangle \log \left(p / p_{0}\right)$ for an ideal-gas atmosphere (gas constant $R$ and reference surface pressure $p_{0}$ ), is proportional to a vertical temperature average $\langle T\rangle$ between the surface and pressure $p$ (Holton 2004, Chapter 1), vertically averaged temperatures in the Tropics can likewise decrease at most quartically with latitude. If temperatures in radiative equilibrium decrease more rapidly than quartically, and, in particular, if they have a nonzero gradient at the equator, a thermally direct meridional circulation must exist to transport heat and to reduce the rate of temperature decrease with latitude, such that Hide's theorem is satisfied. For Earth's atmosphere, in the annual mean, the latitude-dependence of the radiative (or radiative-convective) equilibrium temperature vertically averaged over the troposphere can be approximated by $\left\langle T_{E}\right\rangle \propto \Delta_{b} \cos ^{2} \phi$, with pole-to-equator temperature difference $\Delta_{b}$. In the small-angle approximation, Earth's radiative equilibrium temperature in the troposphere thus decreases quadratically with latitude, so a thermally direct meridional circulation must exist if there is any diffusion of angular momentum. The circulation extends at least to the minimum latitude $\phi_{M}$ at which the equilibrium geopotential $\Phi_{E}=-R\left\langle T_{E}\right\rangle \log \left(p / p_{0}\right)$ satisfies Hide's theorem, $-\partial_{\phi} \Phi_{E} \approx 2 \Omega^{2} a^{2} \phi_{M}^{3}$. This latitude is

$$
\phi_{M}\left(z_{*}\right) \approx\left(\frac{g z_{*}}{\Omega^{2} a^{2}} \frac{\Delta_{b}}{T_{0}}\right)^{1 / 2},
$$

where $z_{*}=-\left(R T_{0} / g\right) \log \left(p / p_{0}\right)$ is the log-pressure height and $T_{0}$ a reference temperature. The latitude bound $\phi_{M}\left(z_{*}\right)$ can only be expected to be relevant at heights $z_{*}$ above the planetary boundary layer, where gradient-wind balance can be assumed to hold. Poleward of the latitude $\phi_{M}$, radiative equilibrium satisfies Hide's theorem (cf. Held \& Hou 1980). A pole-to-equator temperature difference in radiative equilibrium of $\Delta_{h} / T_{0} \approx 80 \mathrm{~K} / 295 \mathrm{~K}$, with Earth's values for the other parameters, gives a minimum circulation extent at $z_{*}=15 \mathrm{~km}$ of $\phi_{M}=25^{\circ}$, similar to the meridional extent of Earth's Hadley circulation (Figure 1c). However, Earth's Hadley circulation above the planetary boundary layer does not exhibit the $\sqrt{z_{*}}$-dependence of meridional extent on height that these considerations would suggest.

Building on the work of Schneider (1977), Held \& Hou (1980) offered a theory of steady axisymmetric circulations in idealized atmospheres, in the "nearly inviscid" limit in which angular momentum diffusion above a planetary boundary layer approaches zero. Angular momentum diffusion above the planetary boundary layer is assumed to be nonzero, so that a meridional circulation exists, but is assumed to be so weak that the zonal wind in the upper branches of the circulation is approximately the angular momentum-conserving wind $u_{M}$. Held \& Hou obtain the meridional extent and strength of the circulation by requiring that the circulation is energetically closed, with diabatic warming in the ascending branches balancing diabatic cooling in the descending branches, and by requiring that temperatures within the circulation make a continuous transition to radiative equilibrium temperatures poleward of it.
Nearly inviscid axisymmetric limit: limit of axisymmetric circulations in which viscosity approaches zero in free atmosphere such that angular momentum there is materially conserved 
Modeling diabatic processes as Newtonian relaxation toward a radiative equilibrium temperature with latitude-dependence $\left\langle T_{E}\right\rangle \propto \Delta_{b} \cos ^{2} \phi$ gives a meridional extent of the circulation that, in the small-angle approximation, scales with the minimum extent $\phi_{M}\left(H_{t}\right)$ at the height $H_{t}$ of the upper branches of the circulation near the tropopause, a height that is assumed to be constant. The strength of the circulation (extremum of mass flux streamfunction) scales as

$$
\left|\Psi_{M}\right| \sim \rho_{0} \frac{a^{2} H_{t}}{\tau}\left(\frac{g H_{t}}{\Omega^{2} a^{2}} \frac{\Delta_{b}}{T_{0}}\right)^{3 / 2} \frac{\Delta_{b}}{\Delta_{v}},
$$

where $\rho_{0}$ is a reference density, $\tau$ is the Newtonian relaxation timescale, and $\Delta_{v}$ is the gross stability, the effective potential temperature difference between the upper and lower branches of the circulation. Unlike the axisymmetric circulations envisaged by Hadley or by Thomson and Ferrel, the ideal Hadley circulation found by Held \& Hou, depending on values of parameters such as the planetary rotation rate $\Omega$ and radius $a$, does not necessarily span hemispheres but has limited meridional extent, with radiative equilibrium poleward of the circulation. The zonal surface wind is easterly near the equator and westerly near the poleward boundaries of the circulation. Above the westerlies, there are shallow, thermally indirect circulation cells, similar to those envisaged by Thomson and Ferrel, in which diffusion transfers angular momentum downward and Coriolis forces on the poleward near-surface flows balance drag on the surface westerlies. For parameters representative of Earth's Hadley circulation, with $\Delta_{h}=80 \mathrm{~K}, \Delta_{v}=70 \mathrm{~K}, T_{0}=295 \mathrm{~K}, H_{t}=15 \mathrm{~km}, \rho_{0}=1 \mathrm{~kg} \mathrm{~m}^{-3}$, and $\tau=30$ days, the circulation according to Held \& Hou's exact expressions (without making the small-angle approximation and taking nondimensional $O(1)$ factors into account) extends to $29^{\circ}$ latitude, similar to Earth's Hadley circulation, and has a strength of $10 \times 10^{9} \mathrm{~kg} \mathrm{~s}^{-1}$, about an order of magnitude weaker than Earth's annual-mean Hadley circulation (cf. Figure 1c).

Held \& Hou showed with numerical simulations that aspects such as the meridional extent and strength of steady axisymmetric circulations approach the predictions of the nearly inviscid theory with decreasing momentum and potential temperature diffusivities. Only vertical diffusion of momentum and potential temperature, representing vertical small-scale turbulent fluxes, for example, in cumulus clouds, was included in Held \& Hou's numerical model, as in other, similar models (e.g., Schneider \& Lindzen 1977, Schneider 1977). The simulated circulations are unstable with respect to axisymmetric perturbations and become unsteady when diffusivities are less than a critical value, which prevented Held \& Hou and others (e.g., Schneider 1977, Lindzen \& Hou 1988, Fang \& Tung 1999) from obtaining steady circulations in the nearly inviscid limit numerically. For the smallest diffusivities for which steady axisymmetric circulations have been obtained, the circulations (particularly their strength) still depend on diffusivities. Vertical diffusion is generally not negligible near the poleward boundaries of the circulations, where meridional temperature gradients and, with them, vertical zonal wind shears are greatest and meridional velocities are small (Held \& Hou 1980). Diffusion also plays a role in middle and higher latitudes, which are not exactly in radiative equilibrium in the simulations but exhibit weak, diffusive meridional circulations. 
Nearly inviscid axisymmetric circulations represent one limit of atmospheric circulations, which, subsequent to the work of Held \& Hou, has been studied extensively and has illuminated several aspects of tropical circulations. For example, Hou \& Lindzen (1992) and Fang \& Tung (1996) have studied how diabatic heating concentrated in narrow regions representing intertropical convergence zones affects axisymmetric circulations; Satoh (1994) has studied how moist processes interact with and modify axisymmetric circulations; Emanuel (1995) has derived a constraint on nearsurface moist entropy gradients from the constraint on interior geopotential gradients implied by Hide's theorem; Plumb \& Hou (1992) have studied axisymmetric monsoon circulations [see Plumb (2006) for a review]; and Pauluis (2004) has studied how dissipation in the planetary boundary layer interacts with axisymmetric circulations in the free troposphere and affects the structure of cross-equatorial flows near the surface. However, the question is whether nearly inviscid axisymmetric theory accounts for the dependence of Earth's or an Earth-like Hadley circulation on external parameters. It is clear that zonal winds within Earth's Hadley circulation are significantly weaker than the angular momentum-conserving wind, which would be, assuming zero zonal wind at the equator, $92 \mathrm{~m} \mathrm{~s}^{-1}$ at $25^{\circ}$ latitude (cf. Figure 1a). Figure $\mathbf{1} \boldsymbol{d}$ shows that Earth's Hadley cells, especially the cross-equatorial winter cell, advect angular momentum - the nonlinear advection that is essential for angular momentum conservation in the upper branches of the circulation in the nearly inviscid axisymmetric limit. Yet this nonlinear advection is not efficient enough to homogenize angular momentum along streamlines of the circulation above the planetary boundary layer (Figure 1c). In particular, the summer Hadley cell does not displace angular momentum contours strongly away from the vertical; streamlines cross angular momentum contours throughout its upper branch. The seasonal cycle provides further clues about the relevance of nearly inviscid axisymmetric theory for Earth's Hadley circulation.

\subsection{Seasonal Cycle}

Lindzen \& Hou (1988) showed that in the nearly inviscid limit, because of the nonlinearity of the angular momentum-conservation constraint, axisymmetric circulations respond nonlinearly to displacements of the latitude of maximum heating off the equator.

Figure $2 \boldsymbol{a}$ shows the mass fluxes in the upper branches of nearly inviscid axisymmetric circulations for different latitudes $\phi_{0}$ at which the radiative equilibrium temperature toward which temperatures are relaxed is maximal. (Parameters are as in the numerical example in the preceding subsection, but with $\Delta_{b}=60 \mathrm{~K}, \Delta_{v}=30 \mathrm{~K}$, and $H_{t}=12 \mathrm{~km}$ to be comparable with the simulations shown in Figure 3 below.) The poleward extent and strength of the cross-equatorial "winter" cell increase as $\left|\phi_{0}\right|$ increases, whereas the strength of the "summer" cell decreases. Averaging the circulations for $\phi_{0}$ and $-\phi_{0}$ gives a circulation that is much stronger (by a factor of 15 for $\phi_{0}=6^{\circ}$ ) than the "equinoctial" circulation driven by relaxation toward the averaged radiative equilibrium, which corresponds to $\phi_{0}=0^{\circ}$ (Figure $2 \boldsymbol{b}$ ). Lindzen \& Hou suggested that such a nonlinear amplification of the annually averaged response to seasonally varying thermal driving accounts for the discrepancy between 


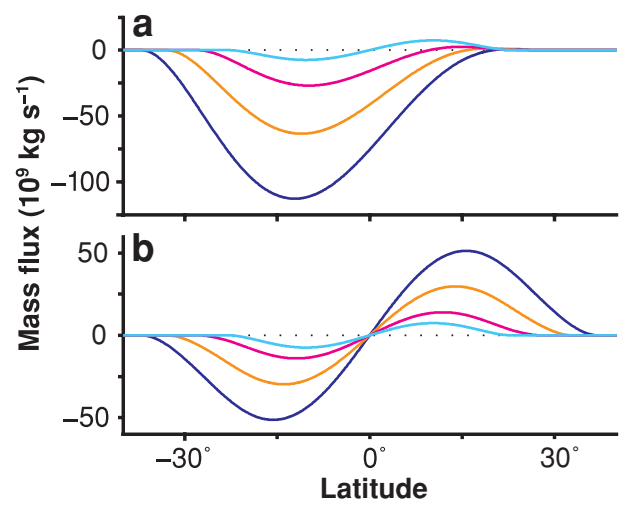

Figure 2

Mass flux (a) and average (e.g., $\phi_{0}=6^{\circ}$ with $\phi_{0}=-6^{\circ}$ ) mass flux $(b)$ in upper branches of nearly inviscid axisymmetric circulations with latitude of maximum radiative equilibrium temperature $\phi_{0}=0^{\circ}$ (light blue), $2^{\circ}$ (magenta), $4^{\circ}$ (orange), and $6^{\circ}$ (blue). Positive values indicate northward flux. From Walker \& Schneider (2005).

the strength of Earth's annual-mean Hadley circulation and the strength of the nearly inviscid axisymmetric circulation driven by relaxation toward an annual-mean radiative equilibrium.

Dima \& Wallace (2003), however, examining the seasonal cycle of Earth's Hadley circulation, found no evidence for nonlinear amplification of the annual-mean circulation. They showed that a large fraction of Earth's Hadley circulation can be attributed to an equinoctial component whose strength does not exhibit the dramatic equinox-solstice swings predicted by nearly inviscid axisymmetric theory. Seasonal variations of the strength of Earth's Hadley circulation are primarily due to a seasonally reversing solstitial component, which is associated with monsoons.

Indeed, nonlinear amplification of the circulation response to seasonally varying thermal driving is already not evident in axisymmetric models with vertical diffusion of momentum and potential temperature, even if the diffusivities are close to the smallest diffusivities for which steady circulations can be obtained.

The top row of Figure 3 shows simulations with an axisymmetric model, similar to the models used by Held \& Hou (1980) and Lindzen \& Hou (1988). The vertical diffusivities for momentum and potential temperature (dry static energy, more precisely) are chosen to be equal and to be close to the smallest diffusivity for which steady circulations for an equilibrium temperature maximum at latitude $\phi_{0}=6^{\circ}$ could be obtained [see Walker \& Schneider (2005) for details]. Compared with heating centered on the equator $\left(\phi_{0}=0^{\circ}\right)$, the circulation with heating displaced off the equator $\left(\phi_{0}=6^{\circ}\right)$ exhibits a significantly strengthened winter cell and weakened summer cell (compare Figures $3 \boldsymbol{a}$ and $\boldsymbol{b}$ ), although the changes are not as dramatic as predicted by nearly inviscid theory (cf. Figure $2 a$ ). However, the average of the circulations for $\phi_{0}=6^{\circ}$ and $\phi_{0}=-6^{\circ}$ is not nonlinearly amplified but is even slightly weaker than the circulation driven by relaxation to the averaged equilibrium temperature (compare Figures $3 \boldsymbol{a}$ and $\boldsymbol{c}$ and contrast them with Figure $2 \boldsymbol{b}$ ). 

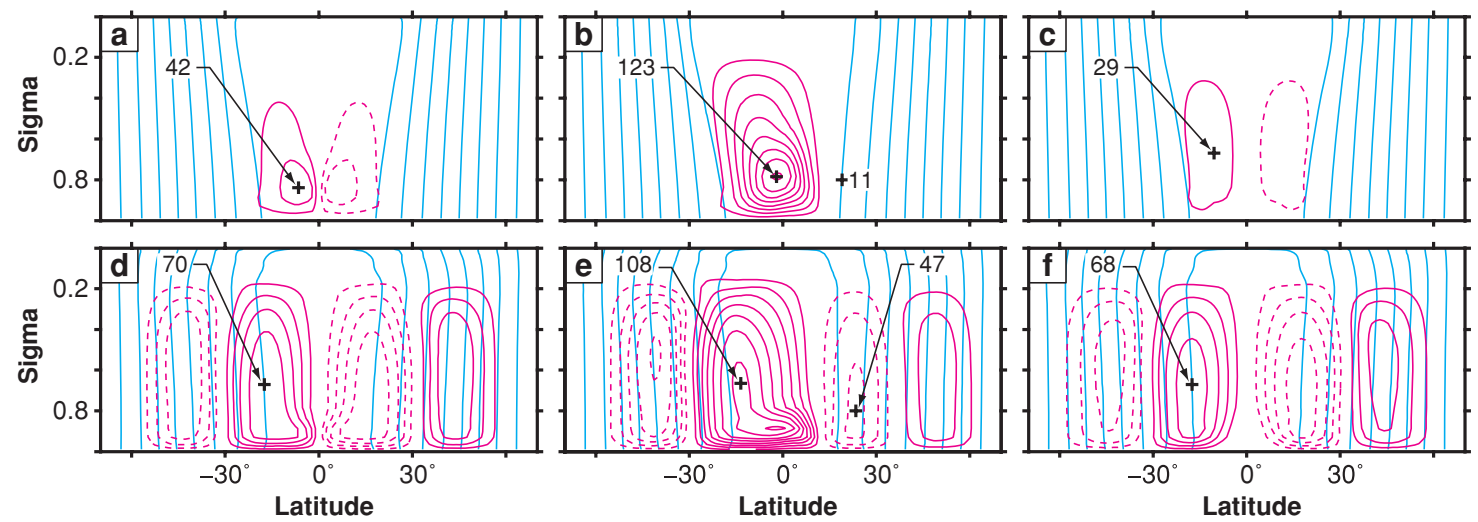

Figure 3

Eulerian mass flux streamfunctions (magenta) and angular momentum (light blue) in axisymmetric (top row) and macroturbulent (bottom row) simulations. ( $a, d)$ Maximum radiative equilibrium temperature on equator $\left(\phi_{0}=0^{\circ}\right) .(b, e)$ Maximum radiative equilibrium temperature north of equator $\left(\phi_{0}=6^{\circ}\right) .(c, f)$ Average of circulations for $\phi_{0}=6^{\circ}$ and $\phi_{0}=-6^{\circ}$. Contour intervals are $15 \times 10^{9} \mathrm{~kg} \mathrm{~s}^{-1}$ for streamfunction and $0.1 \Omega a^{2}$ for angular momentum. Streamfunction extrema above $\sigma=0.85$ are identified. Based on Walker \& Schneider (2005).

The local Rossby number $\operatorname{Ro}=-\zeta / f$, with relative vorticity $\zeta=$ $-(a \cos \phi)^{-1} \partial_{\phi}(u \cos \phi)$, is a nondimensional measure of the smallness of the meridional angular momentum gradient and hence is a measure of proximity to the nearly inviscid limit because the meridional angular momentum gradient is proportional to the absolute vorticity, $f+\zeta=-\left(a^{2} \cos \phi\right)^{-1} \partial_{\phi} M$. The nearly inviscid limit corresponds to $\operatorname{Ro} \rightarrow 1$ such that $f+\zeta=f(1-$ Ro $) \rightarrow 0$ where streamlines are horizontal (in the upper branches of the circulations at latitudes at which vertical velocities vanish). The winter cell in the simulation with $\phi_{0}=6^{\circ}$ is relatively close to the nearly inviscid limit, with local Rossby numbers Ro $\gtrsim 0.9$ in the upper branch indicating that angular momentum is nearly homogenized along streamlines (Figure $3 \boldsymbol{b}$ ). The equinoctial $\left(\phi_{0}=0^{\circ}\right)$ and summer cells, however, are further away from the nearly inviscid limit, with local Rossby numbers in their upper branches of Ro $\lesssim 0.7$ and Ro $\lesssim 0.5$, respectively. Compared with advection, diffusion is relatively more important in the weaker circulation cells. Streamlines cross angular momentum contours near the poleward boundaries of the equinoctial cells and throughout the summer cell (Figure $\mathbf{3} \boldsymbol{a}, \boldsymbol{b}$; streamlines of the summer cell are not visible in the figure, but the streamfunction extremum is identified).

The different degrees to which winter, summer, and equinoctial cells approach the nearly inviscid limit, combined with the dependence of circulation strength on diffusivities even when diffusivities are relatively small, invalidate the predictions of nearly inviscid theory for the relative strength of the cells in the axisymmetric simulations (Walker \& Schneider 2005). To understand how axisymmetric circulations depend on external parameters, one needs to understand the dependence of the circulations on dissipative processes, in particular near the poleward boundaries of the circulations.

Local Rossby number: measures importance of relative vorticity compared with planetary vorticity, or of nonlinear momentum advection compared with Coriolis force 
Baroclinic instability: instability converting available potential energy of mean flow into kinetic energy of perturbation

Available potential energy: sum of potential and internal energy available for conversion to kinetic energy in adiabatic redistribution of mass (Lorenz 1955)

\subsection{Influence of Eddies}

Axisymmetric circulations such as those shown in the top row of Figure 3 are baroclinically unstable [see Pierrehumbert \& Swanson (1995) for a review of baroclinic instability theory]. Nonaxisymmetric perturbations of the circulations grow, first as linear baroclinic waves, which later break and lead to new, macroturbulent statistically steady states in which eddies transport heat, mass, and angular momentum, as well as, in Earth's atmosphere and in more realistic models, water vapor and other tracers. Eddies transport angular momentum into midlatitudes; surface westerlies appear in latitude zones of upper-level angular momentum flux convergence. Eddies also transport heat poleward, reducing meridional temperature gradients, the vertical zonal wind shear, and the strength of the jets at the poleward boundaries of the axisymmetric circulation cells. New jet maxima may appear poleward of the former axisymmetric circulation cells. (Chris Walker has provided an animation of the baroclinic instability of an axisymmetric circulation and of the subsequent spin-up of a macroturbulent circulation. Follow the Supplemental Material link in the online version of this review or at http://www.annualreviews.org/.)

The bottom row of Figure 3 shows the temporal and zonal mean mass flux streamfunction and angular momentum in the statistically steady states of the macroturbulent circulations that correspond to the axisymmetric circulations shown in the top row of the figure. The Hadley cells in the macroturbulent simulations extend further poleward than those in the axisymmetric simulations (cf. Held \& Phillips 1990). (For such comparisons, because the circulations in the axisymmetric simulations do not have sharp poleward boundaries, but weak, diffusive meridional circulations extend into midlatitudes, the latitudes at which absolute values of the mass flux streamfunctions drop to below, say, $10 \%$ of the maximum values should be taken to be the meridional boundaries of the axisymmetric circulations.) Streamlines in the upper branches of the Hadley cells in the macroturbulent simulations cross angular momentum contours, even in the winter cell (near its poleward boundary). In the upper branches of the Hadley cells, the local Rossby numbers Ro $=-\bar{\zeta} / f$, now defined with the temporal and zonal mean relative vorticity $\bar{\zeta}$ and measuring the importance of nonlinear momentum advection by the mean flow relative to the Coriolis force, are reduced in comparison with the axisymmetric simulations. Where streamlines are horizontal, the local Rossby numbers are Ro $\lesssim 0.3$ in the equinoctial cells and summer cell and Ro $\lesssim 0.5$ in the winter cell. Near the poleward boundaries in the upper branches of all cells, in the latitude zones of the descending branches, the local Rossby numbers are $\mathrm{Ro} \approx 0.1$. Eddies strengthen the equinoctial Hadley cells (compare Figures $3 \boldsymbol{a}$ and $\boldsymbol{d}$ ). Eddies also strengthen the summer cell, but they weaken the winter cell (compare Figures $\mathbf{3} \boldsymbol{b}$ and $\boldsymbol{e}$ ). Unlike in nearly inviscid theory for axisymmetric circulations, the mass flux in the Hadley cells in the macroturbulent simulations is of the same order of magnitude as that in Earth's Hadley cells (cf. Schneider 1984, Becker et al. 1997, Kim \& Lee 2001, Walker \& Schneider 2005).

As the local Rossby numbers suggest, nearly inviscid theory for axisymmetric circulations does not provide valid predictions of the response of the Hadley 


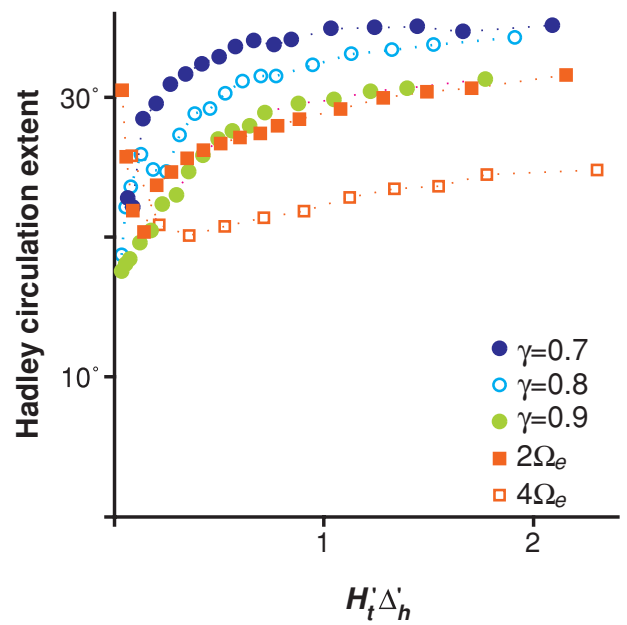

Figure 4

Meridional extent of Hadley circulations in simulations with idealized GCM. Shown are results from simulations with different convective lapse rates $\gamma \Gamma_{d}$ and terrestrial values for other parameters and from simulations with convective lapse rate $0.7 \Gamma_{d}$ but with twice and four times Earth's rotation rate $\Omega_{e}$. For each set of parameters, the figure shows a series of simulations obtained by varying the pole-to-equator surface temperature difference in radiative equilibrium. Adapted from Walker \& Schneider (2006).

circulation to displacements of the latitude of maximum heating. If the latitude of maximum radiative equilibrium temperature is displaced from $\phi_{0}=0^{\circ}$ to $\phi_{0}=6^{\circ}$, the strength of the winter cell increases by a factor of 1.5 and the strength of the summer cell decreases by the same factor (compare Figures $3 \boldsymbol{d}$ and $\boldsymbol{e}$ )—much smaller and more linear changes than predicted by nearly inviscid axisymmetric theory (cf. Figure 2). The average of the circulations for $\phi_{0}=6^{\circ}$ and $\phi_{0}=-6^{\circ}$ is not nonlinearly amplified but is similar to the circulation driven by relaxation to the averaged equilibrium temperature (compare Figures $3 d$ and $f$ ). Inasmuch as they cause departures from angular momentum conservation along streamlines and thus invalidate the predictions of nearly inviscid axisymmetric theory, largescale eddies in macroturbulent simulations play a role similar to diffusion in axisymmetric simulations (Walker \& Schneider 2005); however, their effects generally cannot be modeled as diffusion of angular momentum and/or potential temperature.

The parameter dependence of the meridional extent and strength of Hadley circulations in macroturbulent simulations also differs from that predicted by nearly inviscid axisymmetric theory. Figure 4 shows the extent of Hadley circulations simulated with an idealized GCM as function of the nondimensional parameter $H_{t}^{\prime} \Delta_{b}^{\prime}=\left(H_{t} / H_{0}\right)\left(\Delta_{b} / T_{0}\right)$ appearing in the nearly inviscid axisymmetric scaling estimate $\phi_{M}\left(H_{t}\right)$ (cf. Equation 1). The constant reference height for nondimensionalization is taken to be $H_{0}=12 \mathrm{~km}$, and $T_{0}$ is taken to be the mean 
surface temperature in radiative equilibrium. ${ }^{2}$ The idealized GCM is driven by Newtonian relaxation of temperatures toward radiative equilibrium temperatures that are hemispherically symmetric, maximal at the equator, and statically unstable in the lower troposphere. If an atmospheric column is less stable than a column with temperature lapse rate $\gamma \Gamma_{d}$, where $\gamma$ is a rescaling parameter and $\Gamma_{d}=9.8 \mathrm{~K} \mathrm{~km}^{-1}$ is the dry adiabatic lapse rate, a convection scheme relaxes temperatures toward a profile with lapse rate $\gamma \Gamma_{d}$. The lapse rate in the interior of the simulated Hadley circulations is approximately equal to the convective lapse rate $\gamma \Gamma_{d}$-that is, convection is in "quasi-equilibrium" with its large-scale environment, and horizontal temperature gradients are weak in the interior of the Hadley circulations (Emanuel et al. 1994). Except for the convection scheme mimicking the stabilization of the thermal stratification by moist convection, moist processes are not taken into account in the GCM. Figure 4 shows series of simulations with convective lapse rates between $0.7 \Gamma_{d}$ and $0.9 \Gamma_{d}$ and with planetary rotation rates twice and four times that of Earth. For each series of simulations, the pole-to-equator surface temperature difference in radiative equilibrium $\Delta_{b}$ was varied from $15 \mathrm{~K}$ to $360 \mathrm{~K}$ [see Schneider \& Walker (2006) for a description of the simulations and Walker \& Schneider (2006) for analyses of the Hadley circulations]. The Hadley circulation extent generally does not scale as $\left(H_{t}^{\prime} \Delta_{b}^{\prime}\right)^{1 / 2}$ as nearly inviscid axisymmetric theory would suggest (cf. Equation 1). [There are differences between the thermal driving in the GCM and in the model of Held \& Hou (1980), such as the presence of a convection scheme and a radiative relaxation time that varies spatially near the surface; however, these differences are not what is primarily responsible for the different scaling behavior of the Hadley circulation in the simulations and in nearly inviscid axisymmetric theory (Walker \& Schneider 2006).] The Hadley circulation extent increases relatively rapidly for smaller $H_{t}^{\prime} \Delta_{b}^{\prime}$ and is nearly constant for larger $H_{t}^{\prime} \Delta_{b}^{\prime}$. The Hadley circulation extent decreases with planetary rotation rate, but more slowly than the $\Omega^{-1}$-dependence nearly inviscid axisymmetric theory would suggest. The Hadley circulation extent increases with decreasing convective lapse rate, whereas nearly inviscid axisymmetric theory suggests it would be independent of lapse rates (there would only be an indirect dependence via the dependence of the height $H_{t}$ on lapse rate). The nonmonotonic dependence of Hadley circulation extent on $H_{t}^{\prime} \Delta_{b}^{\prime}$ for small $H_{t}^{\prime} \Delta_{b}^{\prime}$ in the simulations with increased planetary rotation rate is caused by the merging of subtropical and midlatitude jets in circulation regimes with multiple jets in each hemisphere (Walker \& Schneider 2006).

Figure 5 shows the strength of the Hadley circulations in the simulations, expressed in terms of the potential temperature flux at the latitude of the extremal meridional mass flux. According to nearly inviscid axisymmetric theory (Equation 2), the potential temperature flux should scale as $\Delta_{v} \Psi_{M} \sim\left(H_{t}^{\prime} \Delta_{b}^{\prime}\right)^{5 / 2}$. However, the potential temperature flux exhibits two scaling regimes, one for small $H_{t}^{\prime} \Delta_{b}^{\prime}$, in which

\footnotetext{
${ }^{2}$ The temperature $T_{0}$ might also be taken to be the mean temperature or potential temperature of the tropical troposphere for a more direct comparison with Equation 1 or with Held \& Hou (1980); however, in the simulations, these temperatures scale approximately linearly with the (varying) mean surface temperature in radiative equilibrium, so these other choices of $T_{0}$ do not affect scaling laws (Walker \& Schneider 2006).
} 


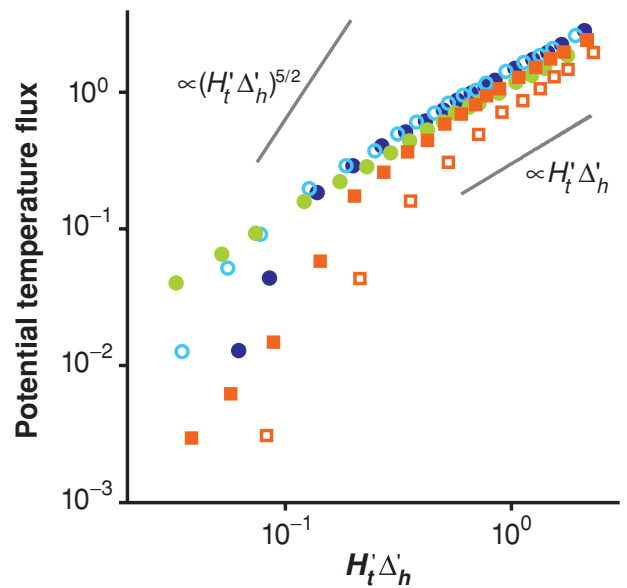

Figure 5

Potential temperature flux (nondimensionalized) in Hadley circulations simulated with idealized GCM. Shown is the potential temperature flux at the latitude of extremal meridional mass flux, vertically integrated over the atmosphere and nondimensionalized such that a flux of one nondimensional unit, if multiplied by the specific heat at constant pressure, corresponds to a poleward energy flux of 4.8 PW. Simulations and plotting symbols as in Figure 4 .

Adapted from Walker \& Schneider (2006).

the scaling of the flux depends on convective lapse rates, among other parameters, and one for large $H_{t}^{\prime} \Delta_{b}^{\prime}$, in which the scaling of the flux is approximately independent of convective lapse rates and is roughly linear (or of a weaker power) in $H_{t}^{\prime} \Delta_{b}^{\prime}$. The potential temperature flux also does not exhibit the $\Omega^{-3}$-dependence on planetary rotation rate that nearly inviscid axisymmetric theory (Equation 2) would suggest [see Walker \& Schneider (2006) for details and additional results].

One can rationalize some aspects of the parameter dependence of the meridional extent and strength of the simulated Hadley circulations by considering the influence of large-scale eddies. The meridional extent of Hadley circulations may be limited by baroclinic instability. If one takes as a heuristic stability criterion for the Hadley circulation that a vertical average of the meridional temperature gradient must not exceed the critical temperature gradient for baroclinic instability in the quasigeostrophic two-layer model, $-a^{-1} \partial_{\phi}\langle T\rangle_{c}=(\beta / f) \Delta_{v}$ with $\beta=a^{-1} \partial_{\phi} f$, an angular momentum-conserving circulation with $u=u_{M}$ for which the geopotential satisfies $-\partial_{\phi} \Phi \approx 2 \Omega^{2} a^{2} \phi^{3}$ in the small-angle approximation, with $\Phi=g z_{*}\langle T\rangle / T_{0}$, is baroclinically unstable poleward of the latitude (cf. Held 2000):

$$
\phi_{c}\left(z_{*}\right) \approx\left(\frac{g z_{*}}{\Omega^{2} a^{2}} \frac{\Delta_{v}}{T_{0}}\right)^{1 / 4}
$$

Using the stability criterion for the two-layer model is a heuristic because there is no critical temperature gradient for baroclinic instability in a continuously stratified atmosphere; however, this heuristic can be made more precise using the supercriticality 
concept discussed in Section 3.2, resulting in the same scaling behavior of the latitude $\phi_{c}$. If the meridional extent of the Hadley circulation is limited by baroclinic instability near the latitude $\phi_{c}\left(H_{t}\right)$, it does not depend on $\Delta_{b}^{\prime}$ but, unlike the latitude $\phi_{M}\left(H_{t}\right)$ at which radiative equilibrium satisfies Hide's theorem, it increases with increasing gross stability $\Delta_{v}$ (or with decreasing lapse rate) near the poleward boundary of the circulation. This parameter dependence is qualitatively consistent with the simulations for larger $H_{t}^{\prime} \Delta_{b}^{\prime}$ shown in Figure 4. One might think that the Hadley circulation extent is determined by the smaller of the two latitude scales $\phi_{c}$ and $\phi_{M}$, so that the scale $\phi_{M}$ is relevant for smaller meridional temperature contrasts and the scale $\phi_{c}$ for larger meridional temperature contrasts (Held 2000). However, these latitude scales do not account quantitatively for the parameter dependence of the Hadley circulation extent (e.g., on planetary rotation rate) in the simulations, for the upper branches of the circulations are not close to the nearly inviscid axisymmetric limit that underlies both scales (i.e., local Rossby numbers are significantly less than one).

A further indication of the influence of eddies on the Hadley circulation is the parameter dependence of the circulation strength. The strength of the Hadley circulations in the simulations is related to the divergence of eddy momentum fluxes. In the temporal and zonal mean, the zonal momentum balance (or, equivalently, the absolute angular momentum balance) in the upper atmosphere, away from the planetary boundary layer, can be approximated by

$$
(f+\bar{\zeta}) \bar{v}=f(1-\operatorname{Ro}) \bar{v} \approx S,
$$

where $v$ is the meridional velocity, $S$ is the eddy momentum flux divergence, and overbars denote temporal and zonal means at constant pressure. We have neglected the vertical advection of zonal momentum, which is generally small in the Tropics of Earth's atmosphere and vanishes at the latitude of the meridional streamfunction extremum, where mean vertical velocities vanish. Over a wide range of the simulations included in Figure 5, the mean meridional velocity at the latitude of the streamfunction extremum and with it the Hadley circulation strength scale as $\bar{v} \sim f^{-1} S$, that is, to the extent that the circulation extent is approximately constant so that the relevant value of the Coriolis parameter is approximately constant, they scale with the eddy momentum flux divergence $S$ in the upper branches of the Hadley cells (Walker \& Schneider 2006). The transition in scaling laws evident in Figure 5, from a scaling law that depends on convective lapse rates to a scaling law that does not, is a transition in scaling laws for eddy momentum fluxes. Eddy momentum fluxes undergo a transition in scaling laws from a regime in which the thermal stratification throughout the atmosphere is set by radiation and convection to a regime in which large-scale eddies influence the thermal stratification in the extratropics and near the poleward boundaries of the Hadley circulation (see Section 3.2). The scaling of the Hadley circulation strength in the simulations is closer to the limit $\mathrm{Ro} \rightarrow 0$, in which the Hadley circulation strength depends on the eddy momentum flux divergence, than to the nearly inviscid axisymmetric limit $\mathrm{Ro} \rightarrow 1$, in which the Hadley circulation strength depends directly on the thermal driving. In the simulations, changes 
in Hadley circulation strength in response to changes, for example, in the pole-toequator temperature gradient in radiative equilibrium, are mediated by changes in eddy momentum flux divergence. This underscores the importance of eddy momentum fluxes, which cause departures from the nearly inviscid axisymmetric limit. If only eddy heat fluxes were important, they could be incorporated in nearly inviscid axisymmetric theory by modifying the thermal driving of the axisymmetric circulations (cf. Schneider 1984). Eddy heat fluxes depend nonlinearly on mean fields (in particular, mean potential temperature gradients) and thus on the mean circulation, so incorporating them in nearly inviscid axisymmetric theory would introduce an additional nonlinearity, but it would not invalidate the basic premises of the theory.

We have theories for Hadley circulations in the nearly inviscid axisymmetric limit $\mathrm{Ro} \rightarrow 1$, in which nonlinear angular momentum advection by the mean flow in the upper branches of the circulations is important, and the Hadley circulation strength responds directly to changes in thermal driving (e.g., Schneider 1977, Held \& Hou 1980, Lindzen \& Hou 1988, Satoh 1994, Fang \& Tung 1996, 1999). We also have theories for Hadley circulations in the limit $\mathrm{Ro} \rightarrow 0$, in which nonlinear angular momentum advection by the mean flow in the upper branches of the circulations is unimportant, and the Hadley circulation strength responds to changes in thermal driving via changes in the thermal structure and in eddy momentum fluxes (e.g. Dickinson 1971, Schneider \& Lindzen 1976, 1977, Fang \& Tung 1994). To account both for the displacement of angular momentum contours away from the vertical, for example, in Earth's winter Hadley cell (Figure 1c), and for the lack of a strongly nonlinear response of the Hadley circulation strength to seasonal variations in thermal driving (Dima \& Wallace 2003), we need a theory of Hadley circulations with local Rossby numbers between the limiting cases of zero and one. One part of the theory must account for the structure of the Hadley circulation given the structure of eddy fluxes. Another part of the theory must account for the structure of eddy fluxes given the Hadley circulation and other mean fields. The first part of the theory may be based on the framework of the weak-temperature gradient approximation for tropical dynamics, which allows one to derive properties of Hadley circulations with intermediate local Rossby numbers (Sobel et al. 2001, Polvani \& Sobel 2002). The second part of the theory must be based on a theory of atmospheric macroturbulence, aspects of which are discussed in the next section. We need such a theory to account for the response of Earth's Hadley circulation not only to seasonally varying thermal driving but also, for example, to increases in greenhouse gas concentrations (e.g., Rind 1998, Dai et al. 2001) and to changes in tropical temperatures such as are associated with El Niño (e.g., Hou 1993, 1998, Chang 1995, Robinson 2002). A theory linking the Hadley circulation and eddy fluxes in idealized dry atmospheres with axisymmetric circulation statistics is a prerequisite for more complete theories that account for statistically nonaxisymmetric circulation features and moist processes, such as monsoon circulations, cloud-radiative feedbacks, or influences of the circulation on the convective stability of the atmosphere through its effect on the distribution of water vapor. 


\section{THE EXTRATROPICAL CIRCULATION}

Isentropic coordinates: coordinate system with entropy or potential temperature as vertical coordinate

\subsection{Isentropic Mass Circulation and Eddy Fluxes}

In the extratropics, the bulk of the transport of heat, mass, angular momentum, and of tracers such as water vapor is effected by large-scale eddies. If one disregards moist convection and latent heat release in large-scale phase changes of water-processes whose role in extratropical dynamics is poorly understood-large-scale eddies are only weakly influenced by diabatic processes because radiative damping timescales are a factor of 5-20 greater than eddy turnover timescales. As already recognized by Shaw (1930, Chapter 6), it is therefore convenient to analyze the extratropical circulation in isentropic coordinates, in which air parcel displacements in large-scale eddies approximately follow horizontal coordinate surface.

In isentropic coordinates, one can generalize many relations known from quasigeostrophic theory without making assumptions such as that variations of the Coriolis parameter or fluctuations of the static stability about a fixed reference value be smallassumptions central to quasigeostrophic theory that are likely not adequate in studies of the general circulation. With the development of the theory of baroclinic instability (Charney 1947, Eady 1949) and the first numerical demonstration of how large-scale eddies affect the general circulation (Phillips 1956), to name two examples, quasigeostrophic theory has led to some of the most fundamental insights in atmospheric dynamics in the twentieth century. In the 1980s, the realization that relations from quasigeostrophic theory can be generalized in isentropic coordinates without making restrictive quasigeostrophic assumptions, largely because quasigeostrophic dynamics can be viewed as representing dynamics on isentropes (Charney \& Stern 1962), led to renewed interest in analyzing the extratropical circulation and especially eddymean flow interactions in isentropic coordinates (e.g., Andrews 1983, Hoskins et al. 1985, Tung 1986, Johnson 1989, Hoskins 1991). The dynamics of the circulation near the lower boundary, which is a fluctuating boundary in isentropic coordinates because the surface potential temperature fluctuates, and their correspondence to quasigeostrophic dynamics have recently become clearer (Held \& Schneider 1999, Koh \& Plumb 2004, Schneider 2005). We are now in a position to analyze the extratropical circulation in isentropic coordinates, relate it to eddy fluxes of quantities that are materially conserved in adiabatic and inviscid air parcel displacements, and address questions not amenable to quasigeostrophic theory, such as the question of what controls the extratropical static stability, a fixed parameter in quasigeostrophic theory.

The mean mass flux along and across isentropes forms hemispheric meridional circulations (Figure 6). Upward and downward fluxes across isentropes imply diabatic warming and cooling. The divergence of cross-isentropic fluxes is balanced by the convergence of along-isentropic fluxes, and vice versa. To the extent that air parcel displacements in large-scale eddies approximately follow isentropes, the isentropic mass flux streamfunction is approximately the Lagrangian mass flux streamfunction. Isentropic mass flux streamlines are approximately mean Lagrangian trajectories of air masses. The meridional mass flux along isentropes represented by the isentropic 


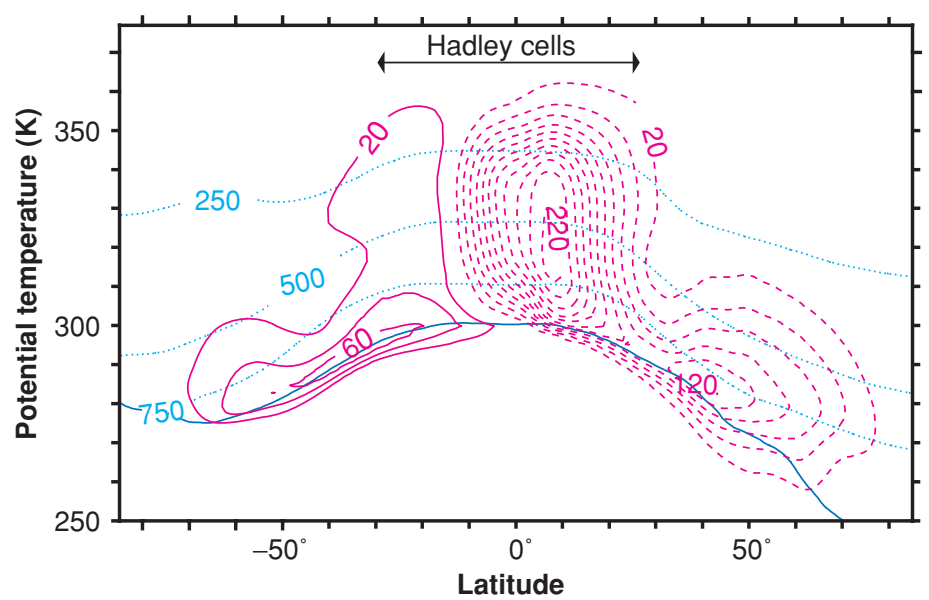

Figure 6

Isentropic mass flux streamfunction for January (temporal and zonal mean according to the same reanalysis data as in Figure 1). Negative streamfunction values (dashed magenta contours) correspond to clockwise rotation, positive values (solid magenta contours) to counterclockwise rotation. The contour interval is $20 \times 10^{9} \mathrm{~kg} \mathrm{~s}^{-1}$. The solid blue line is the median surface potential temperature. The dotted blue lines are mean pressure contours $(250,500$, and $750 \mathrm{hPa})$

streamfunction transports entropy: The meridional entropy flux is the mass flux multiplied by specific entropy, a constant along isentropes. The tropospheric meridional flux of (dry) entropy (or potential temperature) at any given latitude is the tropospheric extremum of the isentropic mass flux streamfunction multiplied by the gross stability, the effective specific entropy (or potential temperature) difference between the poleward upper branch and the equatorward lower branch of the circulation. It is evident that the circulation shown in Figure $\mathbf{6}$ transports entropy poleward. Like the circulation envisaged by Hadley, it is thermally direct and hemispheric, but unlike Hadley's circulation, at least outside the Tropics, it cannot be understood as an axisymmetric circulation with an angular momentum-conserving upper branch. That the isentropic mass circulation in the extratropics is primarily an aggregate of eddy motions is obvious when one compares it with the Eulerian mean mass circulation (compare Figure 6 with Figure 1c): The direction of the isentropic mass flux in midlatitudes is opposite to that of the Eulerian mean mass flux in the Ferrel cells. This also implies that, in the extratropics, the eddy entropy flux dominates the mean entropy flux associated with the Ferrel cells.

The mass flux along isentropes in the extratropics is associated with eddy fluxes of potential vorticity and of surface potential temperature, the two principal materially conserved quantities in adiabatic and inviscid air parcel displacements in the extratropics (Hoskins et al. 1985). The isentropic mass flux streamfunction is proportional to the vertical (i.e., cross-isentropic) integral of the meridional mass flux along isentropes, which is related to eddy fluxes of potential vorticity and of surface potential 
temperature by ${ }^{3}$

$$
\int_{\theta_{b}}^{\theta_{i}} \bar{\rho}_{\theta} \bar{v}^{*} d \theta \approx-\int_{\theta_{b}}^{\theta_{i}} \frac{\bar{\rho}_{\theta} \overline{v^{\prime} \bar{P}^{*}}}{\overline{P^{*}}} d \theta-\bar{\rho}_{\theta}^{0} \overline{v_{g s}^{\prime} \theta_{s}^{\prime}}+M_{E} .
$$

The meridional mass flux along isentropes (left-hand side) is associated with an eddy flux of potential vorticity $P$ along isentropes (first term on right-hand side), an eddy flux of surface potential temperature $\theta_{s}$ (second term), and an Ekman mass flux $M_{E}$ due to zonal frictional forces (third term). The potential temperature $\theta_{b}(\phi)$ is a nominal lower boundary of the integration, smaller than or equal to the lowest surface potential temperature that occurs at the latitude $\phi$ under consideration; the potential temperature $\theta_{i}(\phi)$ is some potential temperature greater than the highest surface potential temperature that occurs at the latitude $\phi$ under consideration. The balance equation (4) is a statement of zonal momentum or potential vorticity balance in the temporal and zonal mean in isentropic coordinates. It holds for circulations with small Rossby numbers, such as in the extratropics of Earth's atmosphere, and generalizes similar expressions known from quasigeostrophic theory; see Schneider (2005) for a derivation, comparisons with quasigeostrophic theory, and a discussion of the conventions on which the balance equation depends. [Tung (1986) and Held \& Schneider (1999) give precursors (incomplete in the treatment of the intersections of isentropes with the surface) and Koh \& Plumb (2004) offer an essentially equivalent variant of the balance equation (4).]

The significance of the balance equation (4) lies in the fact that it relates the isentropic mass circulation and thus entropy fluxes to eddy fluxes of two scalars that are materially conserved in adiabatic and inviscid air parcel displacements. A closure theory for the eddy fluxes of surface potential temperature and of potential vorticity along isentropes must be one central part of any general circulation theory for the extratropics. If the dependence of these eddy fluxes and thus of entropy fluxes on mean fields is known, and if eddies are only weakly influenced by diabatic processes so that correlations between eddy fluctuations and diabatic heating can be neglected in mean-field equations, the thermal structure of the atmosphere is determined by energy-balance requirements, and the vertical zonal wind shear is determined by thermal-wind balance. If the upper boundary of the integration in the balance equation (4) is taken to be the top of the atmosphere $\left(\theta_{i} \rightarrow \infty\right)$, the left-hand side vanishes by mass conservation (cf. Andrews et al. 1987, Chapter 3.8). Upon integration over an atmospheric column, the two eddy-flux terms on the right-hand side balance the Ekman mass flux owing to zonal frictional forces. Therefore, to the extent that zonal frictional forces can be modeled as boundary-layer turbulent dissipation acting on mean zonal winds, the vertical integral of the eddy-flux terms determines the mean

\footnotetext{
${ }^{3}$ Overbars denote temporal and zonal means: $\overline{(\cdot)}$ s along the surface; $\overline{(\cdot)}$ along isentropes if the argument depends on a vertical coordinate; and $\overline{(\cdot)}^{*}=\overline{\left(\rho_{\theta} \cdot\right)} / \bar{\rho}_{\theta}$ along isentropes weighted by the isentropic density $\rho_{\theta}=-g^{-1} \partial_{\theta} p \mathcal{H}\left(\theta-\theta_{s}\right)$, where $p$ is pressure, $\theta$ is potential temperature, and $\mathcal{H}(\cdot)$ is the Heaviside step function. Primes denote fluctuations about the respective means. The subscript $s$ marks surface quantities, $\bar{\rho}_{\theta}^{0}$ is the mean isentropic density at the mean surface potential temperature $\bar{\theta}_{s}$, and $v_{g}$ is the geostrophic meridional velocity.
} 
zonal surface wind [see Green (1970) and Held \& Hoskins (1985) for analogous relations in quasigeostrophic theory]. A theory of the general circulation of dry atmospheres can thus be constructed from a closure theory for the eddy fluxes of surface potential temperature and of potential vorticity along isentropes. Developing a closure theory for eddy fluxes of two scalars that are conserved in adiabatic and inviscid air parcel displacements appears to be easier than developing a closure theory, for example, for eddy entropy fluxes in height-coordinates (vector-valued fluxes) or for eddy momentum fluxes (fluxes of a quantity that is not materially conserved in adiabatic and inviscid displacements). Yet we do not have such a closure theory. We have elements of it, and we have some qualitative understanding of the eddy fluxes.

Qualitatively, the direction of the eddy fluxes can be understood by assuming that eddies mix potential vorticity and surface potential temperature downgradient (Held \& Schneider 1999, Schneider 2005). The isentropic meridional gradient of potential vorticity is generally positive in the interior atmosphere, that is, on isentropes that, at any given latitude, typically do not intersect the surface. In the interior atmosphere, downgradient mixing leads to southward eddy fluxes of potential vorticity along isentropes, which, by balance equation (4), are associated with poleward mass fluxes. The equatorward fluxes necessary to close the isentropic mass circulation occur in the surface layer, that is, on isentropes that, at any given latitude, sometimes intersect the surface (Juckes et al. 1994). In the surface layer, the isentropic meridional gradient of potential vorticity is generally negative-it is related to the surface potential temperature gradient, similar to the potential vorticity gradient in the lower layer of a quasigeostrophic two-layer model (Schneider 2005) —so that downgradient mixing leads to northward eddy fluxes of potential vorticity along isentropes. By balance equation (4), the northward eddy fluxes of potential vorticity are associated with equatorward mass fluxes. Similarly, downgradient mixing implies poleward eddy fluxes of surface potential temperature and so, by balance equation (4), also equatorward mass fluxes. Much of the near-surface equatorward mass flux occurs at potential temperatures less than the median surface potential temperature (Figure 6), that is, in cold air outbreaks. Figure 7 illustrates the isentropic mass circulation and eddy fluxes in the troposphere. The Ekman mass flux owing to zonal frictional forces (the mean mass flux in the lower branch of the Ferrel cell) is poleward in the region of surface westerlies and hence has opposite direction of the mass fluxes associated with the near-surface eddy fluxes.

\subsection{Eddy Fluxes and the Extratropical Thermal Stratification}

A semiempirical quantitative result of how eddy fluxes constrain the thermal stratification of the extratropical troposphere can be obtained under the assumption that eddies mix potential vorticity and surface potential temperature diffusively, with an eddy diffusivity that has no essential vertical structure in the altitude range over which eddies effect significant entropy fluxes, or mass fluxes along isentropes (Schneider \& Walker 2006). The assumption that the eddy diffusivity has no essential vertical structure is justifiable in the limit of strongly baroclinically unstable circulations. In this limit, one would expect the inverse cascade of eddy energy from the scales of baroclinic 


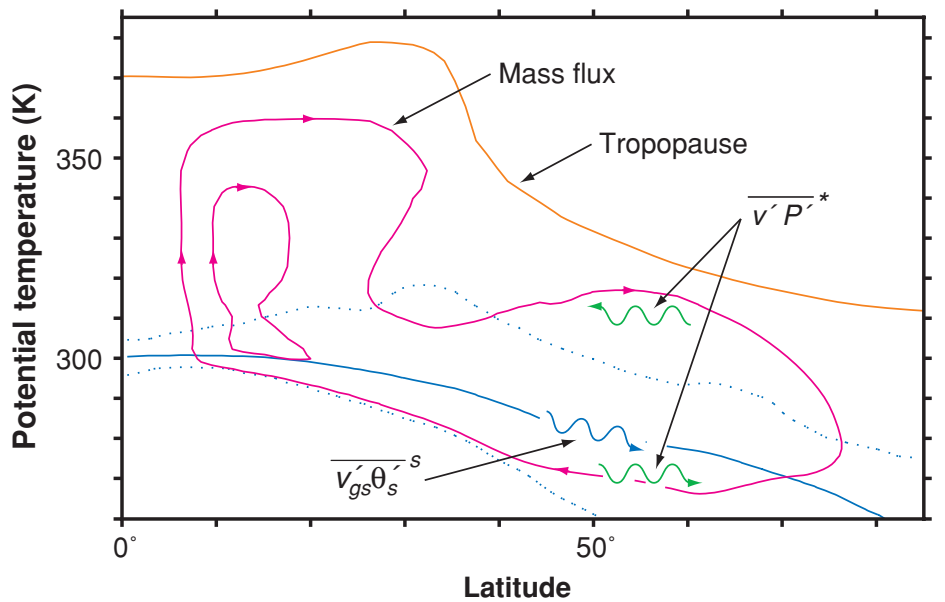

Figure 7

Sketch of isentropic mass circulation and eddy fluxes (based on reanalysis data for the Northern Hemisphere annual mean). Solid magenta lines are contours of the isentropic mass flux streamfunction. Wavy lines indicate eddy fluxes. The solid blue line is the median surface potential temperature (approximately equal to the mean surface potential temperature); the dotted blue lines demarcate the surface layer of isentropes within which, at any given latitude, the instantaneous surface potential temperature lies $90 \%$ of the time.

Inverse energy cascade: turbulent cascade of barotropic eddy kinetic energy from smaller to larger scales

Barotropic kinetic energy: kinetic energy of vertically averaged flow instability to larger horizontal and vertical scales to lead to barotropization of the energy-containing eddies, which dominate the advection of potential vorticity and surface potential temperature fluctuations (e.g., Charney 1971, Salmon 1980, 1982, Held \& Larichev 1996, Smith \& Vallis 2002). For scaling estimates, the assumption that the eddy diffusivity has no essential vertical structure may also be justifiable in weakly baroclinically unstable circulations, provided that, as appears to be the case in Earth's atmosphere, the streamfunction amplitudes of linear or weakly nonlinear baroclinic waves vary only weakly in the altitude range over which the waves effect significant entropy fluxes (cf. Simmons \& Hoskins 1976, 1977, Held 1978, Valdes and Hoskins 1988).

A constraint on the thermal stratification of the extratropical troposphere follows from the balance equation (4) upon substitution of diffusive eddy flux closures ${\overline{v^{\prime}} P^{\prime}}^{*} \sim-\left.D \partial_{y} \bar{P}^{*}\right|_{\theta}$ and $\overline{v_{g s}^{\prime} \theta_{s}^{\prime}} \sim-D \partial_{y} \bar{\theta}_{s}$, with eddy diffusivity $D$ and with latitude coordinate $y=a \phi$. Suppose that, at each latitude, there is a well-defined potential temperature $\theta_{e}$ up to which significant eddy entropy fluxes extend and that above that potential temperature the atmosphere can be assumed to be in radiative or radiativeconvective equilibrium. With the potential temperature $\theta_{e}$ as the upper boundary of the integration, the left-hand side of the balance equation (4) then vanishes by mass conservation. If one ignores the Ekman mass flux (the mean mass flux in the Ferrel cell), substitutes the diffusive eddy flux closures, and uses the assumption that the eddy diffusivity has no essential vertical structure, the eddy diffusivity drops out of the balance equation (4), and one obtains an estimate of the pressure $\bar{p}_{e}=\bar{p}\left(\theta_{e}\right)$ up 
to which significant eddy entropy fluxes extend as a function of mean-field quantities (Schneider \& Walker 2006). [Alternatively, and potentially more generalizable to the circulations of the oceans and of planets without a solid surface, one can assume that the geostrophic component of the isentropic mass circulation closes at potential temperature $\theta_{e}$; see Schneider (2006).] If convective entropy fluxes extend higher than entropy fluxes associated with large-scale eddies, the pressure $\bar{p}_{e}$ will be higher than the pressure $\bar{p}_{t}$ at the tropopause-the upper boundary of the atmospheric layer within which the bulk of the entropy the atmosphere receives at the surface is redistributed (Schneider 2004). If entropy fluxes associated with large-scale eddies extend as high or higher than convective entropy fluxes, large-scale eddies will modify or set the tropopause height, so that the pressure $\bar{p}_{t}$ at the tropopause will be similar to the pressure $\bar{p}_{e}$. Combining the constraint $\bar{p}_{t} \lesssim \bar{p}_{e}$ on tropopause pressure with the estimate of the pressure $\bar{p}_{e}$ obtained from the balance equation (4), one finds that the supercriticality

$$
S_{c}=-\frac{f}{\beta} \frac{\partial_{y} \bar{\theta}_{s}}{\bar{\Delta}_{v}},
$$

with bulk stability $\bar{\Delta}_{v}=-2{\overline{\partial_{p} \theta}}^{s}\left(\bar{p}_{s}-\bar{p}_{t}\right)$, cannot significantly exceed one: $S_{c} \lesssim 1$ (Schneider \& Walker 2006). If the tropopause height is somewhat greater than the scale height, as is the case in Earth's atmosphere (apparently by coincidence), the bulk stability is approximately equal to the potential temperature difference between tropopause and surface, $\bar{\Delta}_{v} \approx \bar{\theta}_{t}-\bar{\theta}_{s}$, similar to the gross stability that appeared in the discussion of the Hadley circulation. The supercriticality is a nondimensional measure of the vertical extent of eddy entropy fluxes relative to the tropopause height, $S_{c} \sim\left(\bar{p}_{s}-\bar{p}_{e}\right) /\left(\bar{p}_{s}-\bar{p}_{t}\right)$, or a nondimensional measure of the slope of near-surface isentropes.

Figure 8 shows bulk stabilities $\bar{\Delta}_{v}$ and scaled surface potential temperature gradients $-(f / \beta) \partial_{y} \bar{\theta}_{s}$ in the macroturbulent idealized GCM simulations already discussed in the context of the Hadley circulation in Section 2.3. Since the supercriticality constraint $S_{c} \lesssim 1$ is based on diffusive eddy flux closures, it can only be expected to hold on scales greater than eddy length scales, so Figure $\mathbf{8}$ shows averages over extratropical baroclinic zones. For small scaled surface potential temperature gradients $-(f / \beta) \partial_{y} \bar{\theta}_{s}$ and for sufficiently small convective lapse rates $\gamma \Gamma_{d}$, bulk stabilities $\bar{\Delta}_{v}$ are approximately independent of the scaled surface potential temperature gradients. In this regime, eddy entropy fluxes are weak, the thermal stratification and tropopause height are set by radiation and convection, and $S_{c}<1$. When scaled surface potential temperature gradients $-(f / \beta) \partial_{y} \bar{\theta}_{s}$ approach the value at which $S_{c} \sim 1$ for a radiativeconvective thermal stratification, bulk stabilities $\bar{\Delta}_{v}$ increase in proportion to the scaled surface potential temperature gradients, such that the supercriticality does not significantly exceed one. In this regime, eddy entropy fluxes stabilize the thermal stratification and modify the tropopause height. The smaller the bulk stability for a radiative-convective thermal stratification (i.e., the larger the convective lapse rate $\left.\gamma \Gamma_{d}\right)$, the smaller is the scaled surface potential temperature gradient $-(f / \beta) \partial_{y} \bar{\theta}_{s}$ at which the line $S_{c} \sim 1$ is reached. For sufficiently large surface potential temperature gradients, all simulations condense onto the line $S_{c} \sim 1$. The simulations span a wide
Supercriticality: nondimensional measure of slope of isentropes or of vertical extent of eddy entropy fluxes 


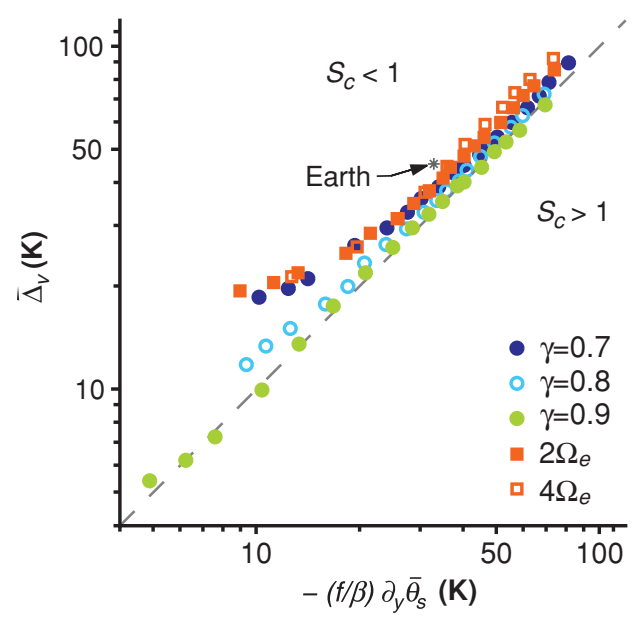

Figure 8

Extratropical bulk stability $\bar{\Delta}_{v}$ and scaled surface potential temperature gradient $-(f / \beta) \partial_{y} \bar{\theta}_{s}$ in idealized GCM simulations. Displayed quantities are averages over extratropical baroclinic zones, for the same simulations as in Figures $\mathbf{4}$ and $\mathbf{5}$. The corresponding quantities for Earth's atmosphere (from reanalysis data for the Northern Hemisphere annual mean) are shown for comparison. The dashed line represents supercriticality $S_{c}=1$, with $S_{c}<1$ above it and $S_{c}>1$ below it. Based on results of Schneider \& Walker (2006).

range of atmospheric circulations, including Earth-like circulations and circulations with multiple jets and regions of surface westerlies in each hemisphere-features such as those seen on the giant planets (Figure $\mathbf{9} \boldsymbol{a}$ and $\boldsymbol{b}$ ).

The supercriticality structurally resembles quasigeostrophic instability measures (e.g., Pedlosky 1970, 1979, Held \& Larichev 1996). For example, a necessary condition for baroclinic instability in the quasigeostrophic two-layer model is that a counterpart of the supercriticality exceeds one. (We used this condition in the discussion of the meridional extent of the Hadley circulation in Section 2.3.) For Earth-like atmospheres, in which the bulk stability is approximately equal to the potential temperature difference between tropopause and surface and in which baroclinic zones are centered in midlatitudes such that $|f / \beta|=|a \tan \phi| \approx a$, a supercriticality $S_{c} \sim 1$ implies $\bar{\theta}_{t}-\bar{\theta}_{s} \sim\left|a \partial_{y} \bar{\theta}_{s}\right|$, which means that the lowest isentrope that crosses the tropopause in high latitudes grazes the surface in low latitudes, as is the case in Earth's atmosphere and in Earth-like atmospheres in the idealized GCM simulations (cf. Figures $1 \boldsymbol{a}$ and $\mathbf{9 a}$ ). That Earth's atmosphere is close to a state with supercriticality one has led to hypotheses that it may be in a baroclinically adjusted state, that is, a state approximately neutral with respect to baroclinic instability (e.g., Stone 1972, 1978, Lindzen \& Farrell 1980, Held 1982, Lindzen 1993, Stone \& Nemet 1996). Although structurally similar to quasigeostrophic baroclinic adjustment constraints, the supercriticality constraint $S_{c} \lesssim 1$ neither presupposes nor implies, however, that the atmosphere is neutral or stable with respect to baroclinic instability.

The supercriticality constraint $S_{c} \lesssim 1$ on the thermal structure of the extratropical troposphere constrains the changes in tropopause height and thermal stratification 

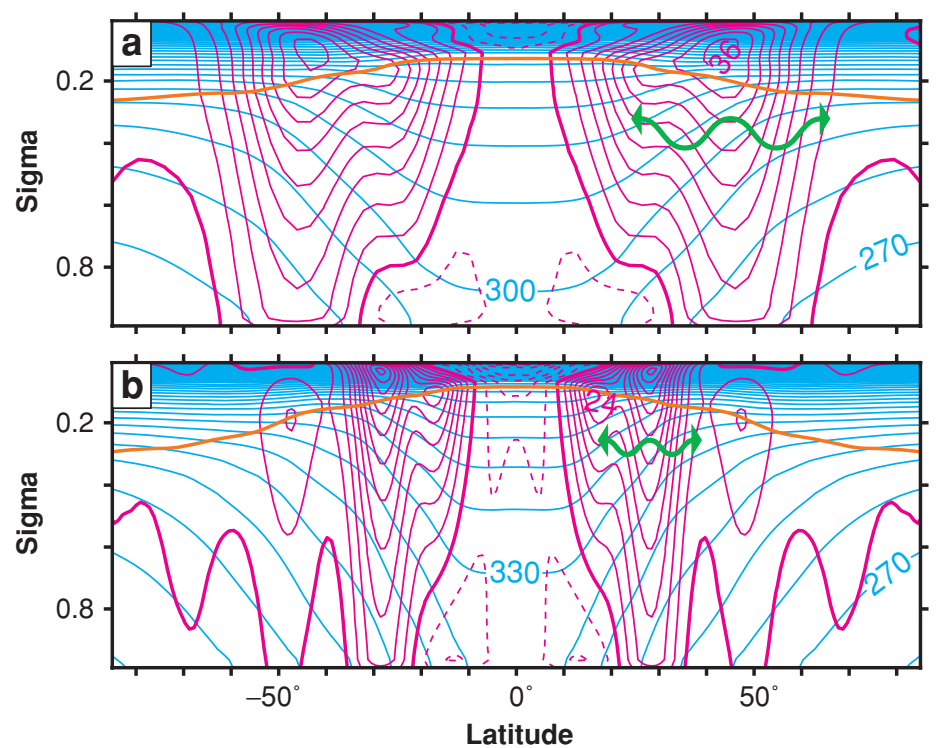

Figure 9

Mean zonal wind (magenta), potential temperature (light blue), tropopause (orange), and eddy length scales (green) in two idealized GCM simulations. As in Figure 1, the contour intervals are $10 \mathrm{~K}$ for potential temperature and $4 \mathrm{~m} \mathrm{~s}^{-1}$ for zonal wind. The meridional extent of the wavy green lines indicates eddy length scales (the lines are centered at the latitude of maximum eddy kinetic energy). (a) Simulation with planet radius and rotation rate of Earth. (b) Simulation with planet radius of Earth and rotation rate four times that of Earth. Adapted from Schneider \& Walker (2006).

that are possible as the climate changes. See Schneider (2006) for a review of how the tropopause height and the thermal stratification are related and can be influenced by eddy dynamics such as those discussed here and by other processes such as moist convection. Evidence that changes in Earth's extratropical tropopause height and thermal stratification in recent decades scale in a manner consistent with $S_{c} \sim 1$ is given by Schneider (2004).

\subsection{Eddy Energies, Eddy Length Scales, and Separation Scales between Jets}

The supercriticality constraint $S_{c} \lesssim 1$ also constrains characteristics of atmospheric macroturbulence. In quasigeostrophic models, circulations with $S_{c} \gg 1$ are possible and have been simulated (e.g., Panetta 1993, Lapeyre \& Held 2003). Quasigeostrophic macroturbulence with $S_{c} \gg 1$ is characterized by strong nonlinear eddy-eddy interactions, which mediate an inverse cascade of barotropic eddy kinetic energy from the scale of the linearly most unstable baroclinic waves to larger scales (Held \& Larichev 1996). Because the thermal stratification is taken to be fixed in quasigeostrophic theory, eddies in quasigeostrophic models cannot stabilize the thermal stratification or 
Figure 10

Eddy available potential energy (EAPE) versus baroclinic eddy kinetic energy $\left(\mathrm{EKE}_{\mathrm{bc}}\right)$. The dashed lines represent the linear relation

$\mathrm{EAPE}=2.25 \mathrm{EKE}_{\mathrm{bc}}$ Simulations and plotting symbols as in Figure 8.

Based on results of Schneider \& Walker (2006).

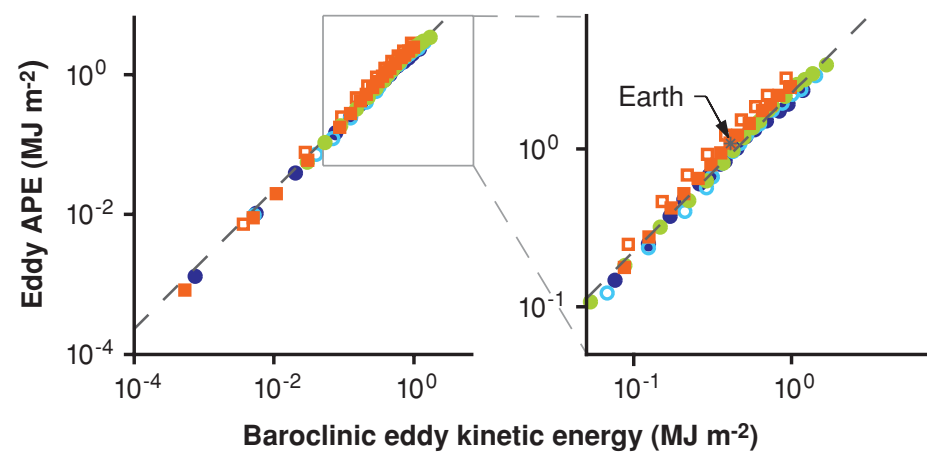

modify counterparts of the tropopause height. In contrast, for more realistic atmospheric macroturbulence, the result that surface potential temperature gradients and the extratropical thermal stratification adjust such that $S_{c} \lesssim 1$ suggests, by analogy to the quasigeostrophic counterparts of the supercriticality, that nonlinear eddy-eddy interactions and the concomitant inverse energy cascade are inhibited.

Perhaps the clearest indication that nonlinear eddy-eddy interactions and an inverse energy cascade beyond the scale of the linearly most unstable baroclinic waves are inhibited comes from eddy energies in the idealized GCM simulations. Because it is nonlinear eddy-eddy interactions that distort the linearly most unstable baroclinic waves in macroturbulent flows, scales and structures of the energy-containing eddies resemble those of the linearly most unstable baroclinic waves if nonlinear eddy-eddy interactions are weak. In particular, eddy available potential energy and baroclinic kinetic energy are equipartitioned in the linearly most unstable baroclinic waves-an equipartitioning that eddy energies in the idealized GCM simulations exhibit clearly (Figure 10). In the simulations, the proportionality constant relating eddy available potential energy and baroclinic eddy kinetic energy appears to depend only on external parameters such as the planetary rotation rate and, not shown in Figure 10, the planet radius (Schneider \& Walker 2006). The ratio of eddy available potential energy and baroclinic eddy kinetic energy in Earth's atmosphere is approximately the same as that in the idealized GCM simulations with terrestrial rotation rate and radius (Figure 10), which may, however, be fortuitous because moist processes not taken into account in the simulations may affect eddy energies in Earth's atmosphere. Eddy available potential energy and barotropic eddy kinetic energy are likewise equipartitioned in the simulations, but this equipartitioning would also be possible if nonlinear eddy-eddy interactions were strong and the inverse energy cascade were significant. If nonlinear eddy-eddy interactions were strong, one would expect eddy available potential energy and barotropic eddy kinetic energy to be greater than baroclinic eddy kinetic energy (by a factor of supercriticality squared in the quasigeostrophic two-layer model), with the ratio of the energies increasing with supercriticality and with the extent of the inverse energy cascade (Held \& Larichev 1996). Such scaling properties are not seen in the idealized GCM simulations, consistent with the supercriticality constraint $S_{c} \lesssim 1$. There may be upscale kinetic energy transfer from 
smaller scales to the scale of the linearly most unstable baroclinic waves, but there is no significant inverse energy cascade beyond it, consistent with analyses of interactions of transient eddies in Earth's atmosphere (Boer \& Shepherd 1983).

Because eddy available potential energy scales as $\operatorname{var}\left(\Psi_{\mathrm{bc}}\right) / L_{R}^{2}$, with Rossby radius $L_{R}$ and baroclinic streamfunction variance var $\left(\Psi_{\mathrm{bc}}\right)$, and baroclinic eddy kinetic energy scales as $\operatorname{var}\left(\Psi_{\mathrm{bc}}\right) / L_{e}^{2}$, with energy-containing scale $L_{e}$, equipartitioning of eddy available potential energy and baroclinic eddy kinetic energy implies that the energy-containing scale is similar to the Rossby radius, as in the linearly most unstable baroclinic waves. This is indeed the case in the idealized GCM simulations (Schneider $\&$ Walker 2006). The energy-containing scale is similar to the Rossby radius, except when the Rossby radius is so large that the energy-containing scale is limited by the finite size of the planet, in which case the energy-containing scale is smaller than the Rossby radius. An examination of length scales likewise does not provide any evidence for a significant inverse energy cascade beyond the scale of the linearly most unstable baroclinic waves.

In the idealized GCM simulations with multiple jets and regions of surface westerlies in each hemisphere, the separation scale between the jets is approximately the energy-containing scale and hence is similar to the Rossby radius. The energycontaining scale at the latitude of maximum eddy kinetic energy for two simulations is indicated in Figure 9. The smaller the energy-containing scale, the more jets fit into each hemisphere, and the more jets form (cf. Panetta 1993, Lee 2005). Larger planets or factors that imply smaller Rossby radii, such as larger planetary rotation rates or smaller static stabilities, lead to more jets and regions of surface westerlies in each hemisphere. The result that the jet separation scale is similar to the Rossby radius is consistent with what is known about Jupiter's circulation: The separation scale between off-equatorial jets and estimates of the Rossby radius are of the same order of magnitude ( $2000 \mathrm{~km}$ ) (Ingersoll \& Kanamori 1995, Ingersoll et al. 2004). However, because Jupiter does not have a solid surface, the theoretical arguments leading to the supercriticality constraint do not directly apply; how they can be generalized is an open question.

These are elements of a closure theory for atmospheric macroturbulence, in which relations between eddy energies, eddy length scales, and the thermal structure of the atmosphere must figure prominently. By reducing surface potential temperature gradients and stabilizing the thermal stratification, atmospheric macroturbulence organizes itself into states with $S_{c} \lesssim 1$ in which nonlinear eddy-eddy interactions are weak. The self-organization of atmospheric macroturbulence into states of weak nonlinear eddy-eddy interactions offers an explanation for the historic successes of linear and weakly nonlinear models in accounting for the scales and structures of largescale eddies in Earth's atmosphere (cf. Simmons \& Hoskins 1976, 1977, Lau 1978, Frederiksen 1983, Valdes and Hoskins 1988). It also suggests that we may be able to use results from weakly nonlinear theories, such as equipartitioning of eddy energies and similarity of eddy length scales and Rossby radii (e.g., Pedlosky 1970, 1979), to develop closure theories, for example, for the eddy fluxes of surface potential temperature and of potential vorticity along isentropes. 


\section{SUMMARY AND OPEN QUESTIONS}

The simulations presented in this review demonstrate that we can simulate broad continua of possible planetary circulations and can infer unambiguous macroscopic scaling laws governing atmospheric circulations. Our understanding of the general circulation, even of the idealized dry atmospheres considered here, is still incomplete. But we have the theoretical concepts (such as available potential energy, Rossby radius, etc.) and computational resources that make it seem possible that a general circulation theory of dry atmospheres, at least on the level of scaling laws, may be within reach.

Incorporating moist processes in a general circulation theory may be considerably more difficult. There are dynamic and kinematic difficulties. Dynamically, the distribution of water vapor in the atmosphere impacts the effective static stability, for example, in the Hadley circulation and in baroclinic eddies. We do not have all necessary theoretical concepts to deal with such moist effects. For example, it is unclear what forms an eddy available potential energy or the scale of the linearly most unstable baroclinic waves take in a moist atmosphere. Kinematically, it is unclear how the distribution of water vapor in the atmosphere is determined in a balance of advection of water vapor and condensation and moistening processes.

\section{SUMMARY POINTS}

1. A tropical Hadley circulation must exist if vertically averaged radiativeconvective equilibrium temperatures decrease faster than quartically with latitude and, in particular, if they have a nonzero gradient at the equator (Hide's theorem).

2. The tropical Hadley circulation is influenced by large-scale eddy fluxes, which reduce local Rossby numbers in the upper branch of the circulation and affect the meridional extent and strength of the circulation, in particular, in the summer cell.

3. The isentropic mass circulation spans hemispheres and is, in the extratropics, associated with eddy fluxes of surface potential temperature and of potential vorticity along isentropes. A qualitative understanding can be gained by assuming that eddies mix potential vorticity and surface potential temperature downgradient.

4. Diffusive eddy flux closures lead to the constraint that the supercriticality $S_{c}$ cannot significantly exceed one. This implies that there is a regime of relatively low baroclinicity, in which radiation and convection maintain the extratropical thermal stratification $\left(S_{c}<1\right)$, and a regime of larger baroclinicity, in which large-scale eddies stabilize the thermal stratification such that $S_{c} \sim 1$. 
5. The supercriticality constraint implies that atmospheric macroturbulence, through feedbacks with the thermal structure of the atmosphere, organizes itself into states of weak nonlinear eddy-eddy interactions, in which there is no significant inverse cascade of eddy energy to scales larger than the scale of the linearly most unstable baroclinic waves.

6. As a consequence of the weakness of nonlinear eddy-eddy interactions, the energy-containing scale and the separation scale between extratropical jets are similar to the scale of the linearly most unstable waves, and eddy kinetic and available potential energies are equipartitioned.

\section{FUTURE ISSUES}

1. What is the strength and meridional extent of the Hadley circulation, given the structure of eddy fluxes?

2. What are the scaling laws of atmospheric macroturbulence, for example, the scaling laws of eddy fluxes of potential vorticity and surface potential temperature, as functions of mean fields such as the mean pole-to-equator surface temperature gradient?

3. What is the meridional and vertical structure of eddy fluxes, given the mean state of the atmosphere? Can weakly nonlinear theories account for the structure of eddy fluxes?

4. How do theories for the Hadley circulation and for atmospheric macroturbulence based on dry dynamics need to be modified in the presence of moist processes, which alter, among other things, the effective static stability of the atmosphere? What is the structure of water vapor fluxes and of the global distribution of water vapor in the troposphere, given statistics of atmospheric macroturbulence?

\section{ACKNOWLEDGMENTS}

Collaborations and conversations with Isaac Held shaped my view of the general circulation and are reflected in this review. Chris Walker and Paul O'Gorman kindly provided simulation results and reanalysis data in a format that helped to produce the figures. I thank Paul O'Gorman, Olivier Pauluis, Adam Sobel, and Geoffrey Vallis for reviewing the manuscript and for offering helpful suggestions. I gratefully acknowledge financial support from the Davidow Discovery Fund, the Alfred P. Sloan Foundation, the National Science Foundation (grant no. 0450059), and the Aspen Center for Physics, where I wrote part of this review. The National Center for Atmospheric Research (which is sponsored by the National Science Foundation) provided computational support and the reanalysis data. 


\section{LITERATURE CITED}

Andrews DG. 1983. A finite-amplitude Eliassen-Palm theorem in isentropic coordinates. F. Atmos. Sci. 40:1877-83

Andrews DG, Holton JR, Leovy CB. 1987. Middle Atmosphere Dynamics. Orlando, FL: Academic Press. 489 pp.

Becker E, Schmitz G, Geprägs R. 1997. The feedback of midlatitude waves onto the Hadley cell in a simple general circulation model. Tellus. 49A:182-99

Boer GJ, Shepherd TG. 1983. Large-scale two-dimensional turbulence in the atmosphere. F. Atmos. Sci. 40:164-84

Chang EKM. 1995. The influence of Hadley circulation intensity changes on extratropical climate in an idealized model. F. Atmos. Sci. 52:2006-24

Charney JG. 1947. The dynamics of long waves in a baroclinic westerly current. $\mathcal{F}$. Meteorol. 4:135-63

Charney JG. 1971. Geostrophic turbulence. F. Atmos. Sci. 28:1087-95

Charney JG, Stern ME. 1962. On the stability of internal baroclinic jets in a rotating atmosphere. F. Atmos. Sci. 19:159-72

Dai A, Wigley TML, Boville BA, Kiehl JT, Buja LE. 2001. Climates of the twentieth and twenty-first centuries simulated by the NCAR climate system model. $\mathcal{F}$. Clim. 14:485-519

Defant A. 1921. Die Zirkulation der Atmosphäre in den gemässigten Breiten der Erde: Grundzüge einer Theorie der Klimaschwankungen. Geograf. Ann. 3:209-66

Dickinson RE. 1971. Analytic model for zonal winds in the Tropics: I. Details of the model and simulation of gross features of the zonal mean troposphere. Mon. Weather Rev. 99:501-10

Dima IM, Wallace JM. 2003. On the seasonality of the Hadley cell. 7. Atmos. Sci. 60:1522-27

Eady ET. 1949. Long waves and cyclone waves. Tellus 1:33-52

Emanuel KA. 1995. On thermally direct circulations in moist atmospheres. F. Atmos. Sci. 52:1529-34

Emanuel KA, Neelin JD, Bretherton CS. 1994. On large-scale circulations in convecting atmospheres. Q. F. R. Meteorol. Soc. 120:1111-43

Fang M, Tung KK. 1994. Solution to the Charney problem of viscous symmetric circulation. 7. Atmos. Sci. 51:1261-72

Fang M, Tung KK. 1996. A simple model of nonlinear Hadley circulation with an ITCZ: analytic and numerical solutions. F. Atmos. Sci. 53:1241-61

Fang M, Tung KK. 1999. Time-dependent nonlinear Hadley circulation. F. Atmos. Sci. 56:1797-807

Ferrel W. 1859. The motion of fluids and solids relative to the Earth's surface. Math. Mon. 1:140-48, 210-16, 300-7, 366-73, 397-406

Frederiksen JS. 1983. Disturbances and eddy fluxes in Northern Hemisphere flows: instability of three-dimensional January and July flows. F. Atmos. Sci. 40:836-55

Green JSA. 1970. Transfer properties of the large-scale eddies and the general circulation of the atmosphere. Q. F. R. Meteorol. Soc. 96:157-85

Hadley G. 1735. Concerning the cause of the general trade-winds. Phil. Trans. 39:5862 
Held IM. 1975. Momentum transport by quasi-geostrophic eddies. F. Atmos. Sci. 32:1494-97

Held IM. 1978. The vertical scale of an unstable baroclinic wave and its importance for eddy heat flux parameterizations. 7. Atmos. Sci. 35:572-76

Held IM. 1982. On the height of the tropopause and the static stability of the troposphere. F. Atmos. Sci. 39:412-17

Held IM. 2000. The general circulation of the atmosphere. Proc. Prog. Geophys. Fluid Dyn. Woods Hole Oceanogr. Inst., Woods Hole, MA, http://gfd.whoi.edu/proceedings/2000/PDFvol2000.html

Held IM, Hoskins BJ. 1985. Large-scale eddies and the general circulation of the troposphere. In Advances in Geophysics. Vol. 28: Issues in Atmospheric and Oceanic Modeling. Part A: Climate Dynamics, ed. S Manabe, pp. 3-31. Orlando, FL: Academic Press

Held IM, Hou AY. 1980. Nonlinear axially symmetric circulations in a nearly inviscid atmosphere. F. Atmos. Sci. 37:515-33

Held IM, Larichev VD. 1996. A scaling theory for horizontally homogeneous, baroclinically unstable flow on a beta-plane. 7. Atmos. Sci. 53:946-52

Held IM, Phillips PJ. 1990. A barotropic model of the interaction between the Hadley cell and a Rossby wave. 7. Atmos. Sci. 47:856-69

Held IM, Schneider T. 1999. The surface branch of the zonally averaged mass transport circulation in the troposphere. 7. Atmos. Sci. 56:1688-97

Hide R. 1969. Dynamics of the atmospheres of the major planets with an appendix on the viscous boundary layer at the rigid bounding surface of an electricallyconducting rotating fluid in the presence of a magnetic field. 7. Atmos. Sci. 26:841-53

Holton JR. 2004. An Introduction to Dynamic Meteorology. Boston, MA: Elsevier. 529 pp. 4th ed.

Hoskins BJ. 1991. Towards a PV- $\theta$ view of the general circulation. Tellus. 43AB:27-35

Hoskins BJ, McIntyre ME, Robertson AW. 1985. On the use and significance of isentropic potential vorticity maps. Q. F. R. Meteorol. Soc. 111:877-946

Hou AY. 1993. The influence of tropical heating displacements on the extratropical climate. 7. Atmos. Sci. 50:3553-70

Hou AY. 1998. Hadley circulation as a modulator of the extratropical climate. $\mathcal{F}$. Atmos. Sci. 55:2437-57

Hou AY, Lindzen RS. 1992. The influence of concentrated heating on the Hadley circulation. 7. Atmos. Sci. 49:1233-41

Ingersoll AP, Dowling TE, Gierasch PJ, Orton GS, Read PL, et al. 2004. Dynamics of Jupiter's atmosphere. In Jupiter: The Planet, Satellites, and Magnetosphere, ed. F Bagenal, TE Dowling, WB McKinnon, pp. 105-28. Cambridge, UK: Cambridge Univ. Press. 748 pp.

Ingersoll AP, Kanamori H. 1995. Waves from the collisions of comet ShoemakerLevy 9 with Jupiter. Nature 374:706-8

Ioannou P, Lindzen RS. 1986: Baroclinic instability in the presence of barotropic jets. F. Atmos. Sci. 43:2999-3014

Jeffreys H. 1926. On the dynamics of geostrophic winds. Q. F. R. Meteorol. Soc. 52:85104
Introduction to general circulation theories (lecture notes).

Introduction to atmospheric dynamics. 
Includes historical overview of general circulation theories.

Introduction to equations of motion and compilation of Earth's circulation statistics.
Johnson DR. 1989. The forcing and maintenance of global monsoonal circulations: an isentropic analysis. In Advances in Geophysics. Vol. 31, ed. B Saltzman, pp. 43-316. San Diego, CA: Academic

Juckes MN, James IN, Blackburn M. 1994. The influence of Antarctica on the momentum budget of the southern extratropics. Q. F. R. Meteorol. Soc. 120:1017-44

Kållberg P, Simmons A, Uppala S, Fuentes M. 2004. The ERA-40 archive. ECMWF Tech. Rep. Eur. Cent. Medium Range Weather Forecasts, Reading, England, http://www.ecmwf.int/publications

Kim HK, Lee SY. 2001. Hadley cell dynamics in a primitive equation model. Part II: nonaxisymmetric flow. 7. Atmos. Sci. 58:2859-71

Koh TY, Plumb RA. 2004. Isentropic zonal average formulation and the near-surface circulation. Q. 7. R. Meteorol. Soc. 130:1631-54

Lapeyre G, Held IM. 2003. Diffusivity, kinetic energy dissipation, and closure theories for the poleward eddy heat flux. F. Atmos. Sci. 60:2907-16

Lau NG. 1978. On the three-dimensional structure of the observed transient eddy statistics of the Northern Hemisphere wintertime circulation. 7. Atmos. Sci. 35:1900-23

Lee S. 2005. Baroclinic multiple zonal jets on the sphere. 7. Atmos. Sci. 62:2484-98

Lindzen RS. 1993. Baroclinic neutrality and the tropopause. F. Atmos. Sci. 50:1148-51

Lindzen RS, Farrell B. 1980. The role of the polar regions in global climate, and a new parameterization of global heat transport. Mon. Weather Rev. 108:2064-79

Lindzen RS, Hou AY. 1988. Hadley circulations for zonally averaged heating centered off the equator. 7. Atmos. Sci. 45:2416-27

Lorenz EN. 1955. Available potential energy and the maintenance of the general circulation. Tellus 7:157-67

Lorenz EN. 1967 The Nature and Theory of the General Circulation of the Atmosphere. Geneva: World Meteorol. Org. 161 pp.

Lorenz EN. 1969. The nature of the global circulation of the atmosphere: a present view. In The Global Circulation of the Atmosphere, ed. GA Corby, pp. 3-23. London: R. Meteorol. Soc.

Lorenz EN. 1983. A history of prevailing ideas about the general circulation of the atmosphere. Bull. Am. Meteorol. Soc. 64:730-34

Panetta RL. 1993. Zonal jets in wide baroclinically unstable regions: persistence and scale separation. 7. Atmos. Sci. 50:2073-106

Pauluis O. 2004. Boundary layer dynamics and cross-equatorial Hadley circulation. 7. Atmos. Sci. 61:1161-73

Pedlosky J. 1970. Finite-amplitude baroclinic waves. F. Atmos. Sci. 27:15-30

Pedlosky J. 1979. Finite-amplitude baroclinic waves in a continuous model of the atmosphere. F. Atmos. Sci. 36:1908-24

Peixoto JP, Oort AH. 1992. Physics of Climate. New York: Am. Inst. Phys. 520 pp.

Phillips NA. 1956. The general circulation of the atmosphere: a numerical experiment. Q. F. R. Meteorol. Soc. 82:123-64

Pierrehumbert RT, Swanson KL. 1995. Baroclinic instability. Annu. Rev. Fluid Mech. 27:419-67 
Plumb RA. 2006. Dynamical constraints on monsoon circulations. See Schneider \& Sobel 2006. Preprint: http://eaps.mit.edu/ rap

Plumb RA, Hou AY. 1992. The response of a zonally symmetric atmosphere to subtropical thermal forcing: Threshold behavior. 7. Atmos. Sci. 49:1790-99

Polvani LM, Sobel AH. 2002. The Hadley circulation and the weak temperature gradient approximation. 7. Atmos. Sci. 59:1744-52

Rind D. 1998. Latitudinal temperature gradients and climate change. F. Geophys. Res. 103D:5943-71

Robinson WA. 2002. On the midlatitude thermal response to tropical warmth. Geophys. Res. Lett. 29:1190, doi:10.1029/2001GL014158

Salmon R. 1980. Baroclinic instability and geostrophic turbulence. Geophys. Astrophys. Fluid Dyn. 15:167-211

Salmon R. 1982. Geostrophic turbulence. In Topics in Ocean Physics, Proc. Int. School of Physics 'Enrico Fermi,' eds. AR Osborne and P Malanotte Rizzoli, pp. 30-78. Amsterdam: North-Holland

Satoh M. 1994. Hadley circulations in radiative-convective equilibrium in an axially symmetric atmosphere. 7. Atmos. Sci. 51:1947-68

Schneider EK. 1977. Axially symmetric steady-state models of the basic state for instability and climate studies. Part II. Nonlinear calculations. 7. Atmos. Sci. 34:280-96

Schneider EK. 1984. Response of the annual and zonal mean winds and temperatures to variations in the heat and momentum sources. F. Atmos. Sci. 41:1093-115

Schneider EK, Lindzen RS. 1976. The influence of stable stratification on the thermally driven tropical boundary layer. F. Atmos. Sci. 33:1301-7

Schneider EK, Lindzen RS. 1977. Axially symmetric steady-state models of the basic state for instability and climate studies. Part I. Linearized calculations. F. Atmos. Sci. 34:263-79

Schneider T. 2004. The tropopause and the thermal stratification in the extratropics of a dry atmosphere. F. Atmos. Sci. 61:1317-40

Schneider T. 2005. Zonal momentum balance, potential vorticity dynamics, and mass fluxes on near-surface isentropes. F. Atmos. Sci. 62:1884-900

Schneider T. 2006. The thermal stratification of the extratropical troposphere. See Schneider \& Sobel 2006. Preprint: http://www.gps.caltech.edu/ ־tapio

Schneider T, Sobel AH, eds. 2006. The Global Circulation of the Atmosphere: Phenomena, Theory, Challenges. Princeton, NJ: Princeton Univ. Press

Schneider T, Walker CC. 2006. Self-organization of atmospheric macroturbulence into critical states of weak nonlinear eddy-eddy interactions. F. Atmos. Sci. 63:In press. Preprint: http://www.gps.caltech.edu/ ־tapio

Shaw N. 1930. Manual of Meteorology. Vol. III. The Physical Processes of Weather. Cambridge, UK: Cambridge Univ. Press. 445 pp.

Simmons AJ, Hoskins BJ. 1976. Baroclinic instability on the sphere: normal modes of the primitive and quasigeostrophic equations. F. Atmos. Sci. 33:1454-77

Simmons AJ, Hoskins BJ. 1977. Baroclinic instability on the sphere: solutions with a more realistic tropopause. F. Atmos. Sci. 34:581-88

Simmons AJ, Hoskins BJ. 1978. The life cycles of some nonlinear baroclinic waves. 7. Atmos. Sci. 35:414-32
Review of quasigeostrophic turbulence theory. 
Smith KS, Vallis GK. 2002. The scales and equilibration of midocean eddies: forceddissipative flow. 7. Phys. Oceanogr. 32:1699-720

Sobel AH, Nilsson J, Polvani LM. 2001. The weak temperature gradient approximation and balanced tropical moisture waves. 7. Atmos. Sci. 58:3650-65

Stone PH. 1972. A simplified radiative-dynamical model for the static stability of rotating atmospheres. F. Atmos. Sci. 29:405-18

Stone PH. 1978. Baroclinic adjustment. 7. Atmos. Sci. 35:561-71

Stone PH, Nemet B. 1996. Baroclinic adjustment: a comparison between theory, observations, and models. F. Atmos. Sci. 53:1663-74

Thomson J. 1857. On the grand currents of atmospheric circulation. In Report of the Meeting of the British Association for the Advancement of Science, pp. 38-39. London: Brit. Assoc. [Abstract of paper given at British Association meeting. Full account of theory given in Thomson (1892).]

Thomson J. 1892. Bakerian Lecture. On the grand currents of atmospheric circulation. Phil. Trans. R. Soc. A 183:653-84

Tung KK. 1986. Nongeostrophic theory of zonally averaged circulation. Part I: formulation. 7. Atmos. Sci. 43:2600-18

Valdes PJ, Hoskins BJ. 1988. Baroclinic instability of the zonally averaged flow with boundary layer damping. F. Atmos. Sci. 45:1584-93

Walker CC, Schneider T. 2005. Response of idealized Hadley circulations to seasonally varying heating. Geophys. Res. Lett. 32:L06813, doi:10.1029/2004GL022304

Walker CC, Schneider T. 2006. Eddy-influences on Hadley circulations: simulations with an idealized GCM. F. Atmos. Sci. Preprint: http://www.gps. caltech.edu/ tapio 